ORNL/LTR-2015/773

OAK RIDGE

ORNL/PTS/60562

NATIONAL LABORATORY

MANAGED BY UT-BATTELLE

FOR THE DEPARTMENT OF ENERGY

\title{
On Line Enrichment Monitor (OLEM) UF 6 Tests for 1.5" Sch40 SS Pipe Revision 1
}

José March-Leuba

Jim Garner

Jim Younkin

Darrell W. Simmons

Oak Ridge National Laboratory

January 2016

Approved for public release.

Distribution is unlimited. 


\section{DOCUMENT AVAILABILITY}

Reports produced after January 1, 1996, are generally available free via the U.S. Department of Energy (DOE) Information Bridge.

Web site http://www.osti.gov/bridge

Reports produced before January 1, 1996, may be purchased by members of the public from the following source.

National Technical Information Service

5285 Port Royal Road

Springfield, VA 22161

Telephone 703-605-6000 (1-800-553-6847)

TDD 703-487-4639

Fax 703-605-6900

E-mail info@ntis.gov

Web site http://www.ntis.gov/support/ordernowabout.htm

Reports are available to DOE employees, DOE contractors, Energy Technology Data Exchange (ETDE) representatives, and International Nuclear Information System (INIS) representatives from the following source.

Office of Scientific and Technical Information

P.O. Box 62

Oak Ridge, TN 37831

Telephone 865-576-8401

Fax 865-576-5728

E-mail reports@osti.gov

Web site http://www.osti.gov/contact.html

This report was prepared as an account of work sponsored by an agency of the United States Government. Neither the United States Government nor any agency thereof, nor any of their employees, makes any warranty, express or implied, or assumes any legal liability or responsibility for the accuracy, completeness, or usefulness of any information, apparatus, product, or process disclosed, or represents that its use would not infringe privately owned rights. Reference herein to any specific commercial product, process, or service by trade name, trademark, manufacturer, or otherwise, does not necessarily constitute or imply its endorsement, recommendation, or favoring by the United States Government or any agency thereof. The views and opinions of authors expressed herein do not necessarily state or reflect those of the United States Government or any agency thereof. 


\section{TABLE OF CONTENTS}

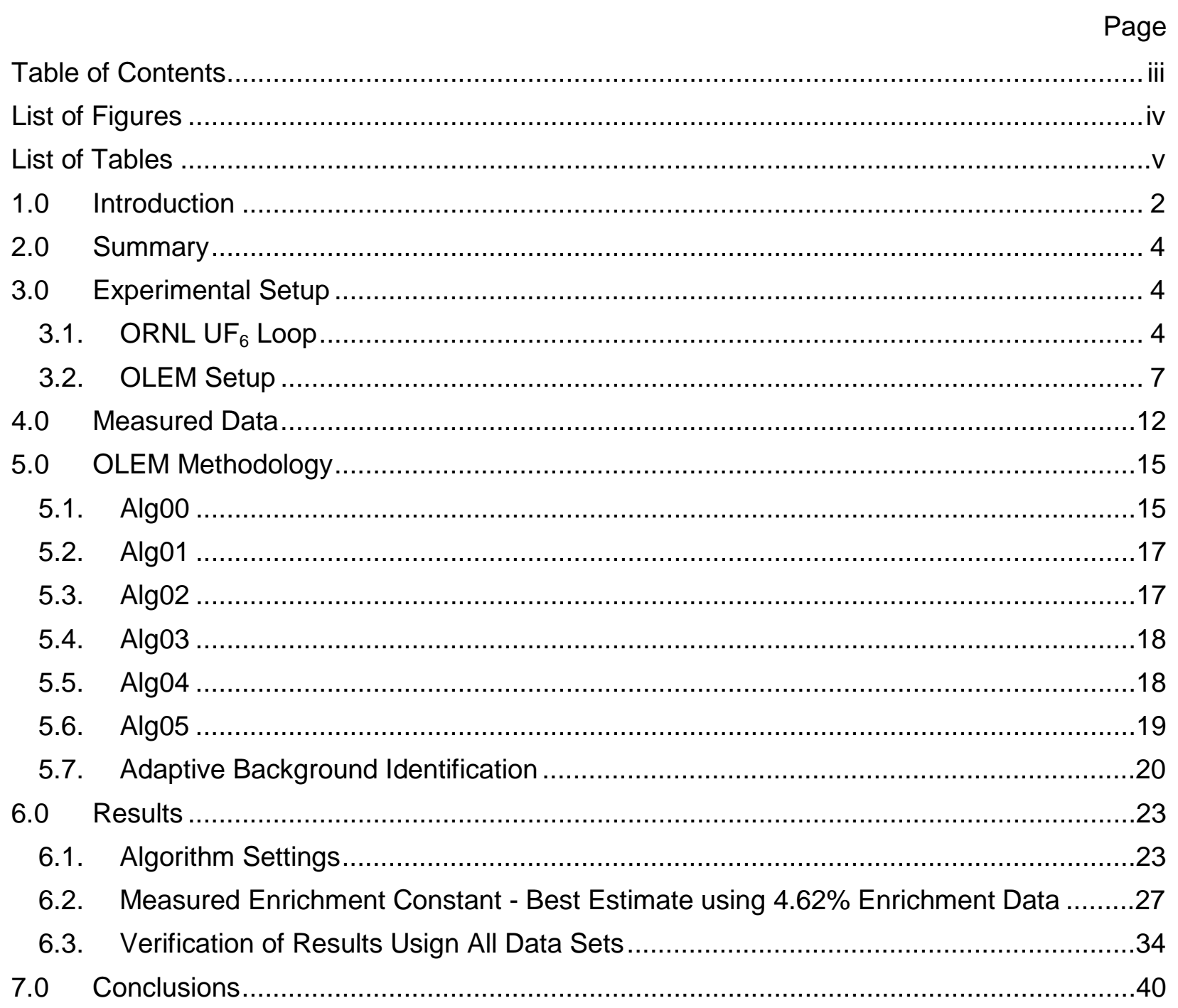




\section{LIST OF FIGURES}

Page

Figure 1. OLEM Collection Node.

Figure 2. Schematic of $U_{6}$ Test Loop \#2 as configured for this testing............................. 5

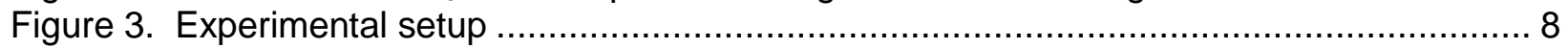

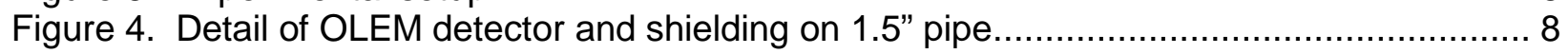

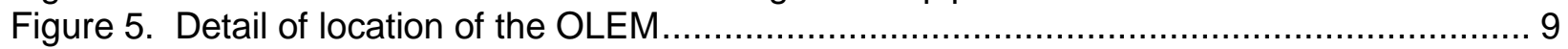

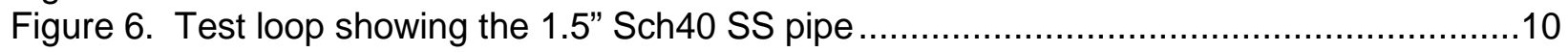

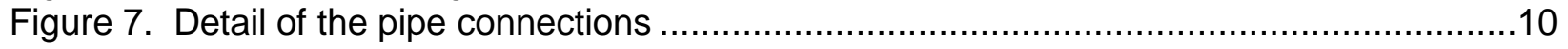

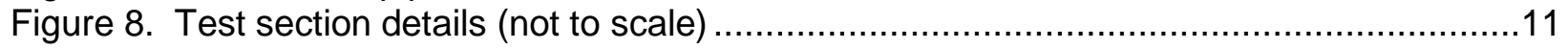

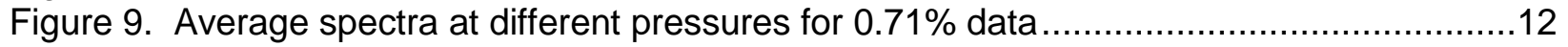

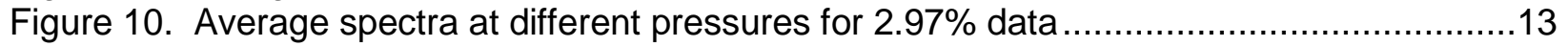

Figure 11. Average spectra at different pressures for $4.62 \%$ data ...................................13

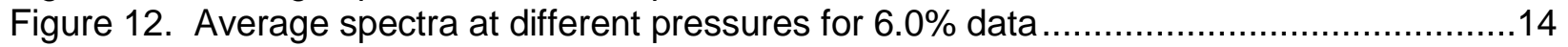

Figure 13. Average spectra at different pressures for $93.7 \%$ data ..................................14

Figure 14. Illustration of OLEM algorithms application to simulated data..............................15

Figure 15. Illustration of Alg00 trapezoidal background removal under the peak...................16

Figure 16. Illustration of Alg01 count rate methodology ...............................................

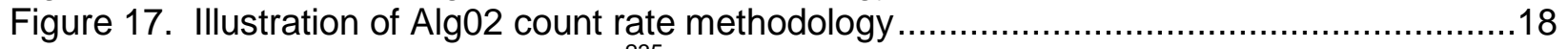

Figure 18. Illustration of Algorithm $05{ }^{235} \mathrm{U}$ spectrum fit process ......................................20

Figure 19. Spectral decomposition for case with sinusoidal background .............................22

Figure 20. Spectral decomposition for case with step background .................................22

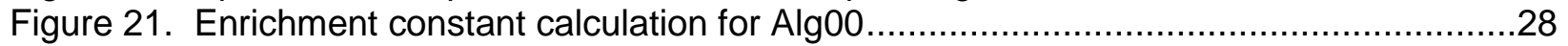

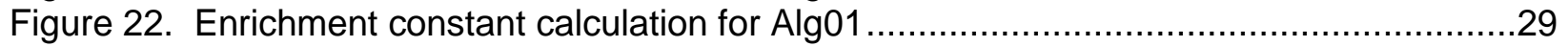

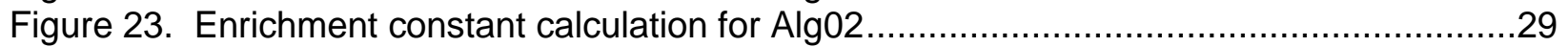

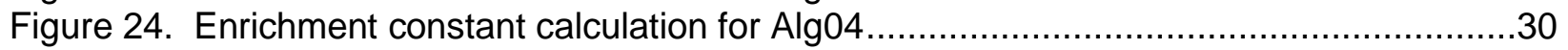

Figure 25. Enrichment constant calculation for Alg05 ................................................

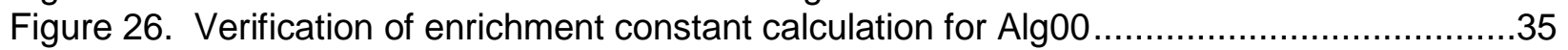

Figure 27. Verification of enrichment constant calculation for Alg01 ..................................35

Figure 28. Verification of enrichment constant calculation for Alg02 ….............................36

Figure 29. Verification of enrichment constant calculation for Alg04 .................................36

Figure 30. Verification of enrichment constant calculation for Alg05 ...............................37

Figure 31. Verification of enrichment constant calculation for Alg00, including the 93.7\% data

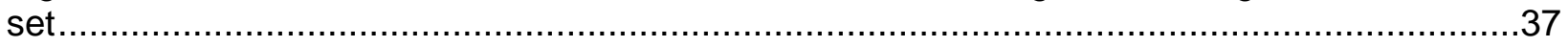

Figure 32. Verification of enrichment constant calculation for Alg01, including the $93.7 \%$ data

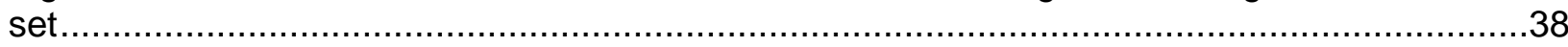

Figure 33. Verification of enrichment constant calculation for Alg02, including the $93.7 \%$ data set.

Figure 34. Verification of enrichment constant calculation for Alg04, including the $93.7 \%$ data

set.

Figure 35. Verification of enrichment constant calculation for Alg05, including the $93.7 \%$ data set. 


\section{LIST OF TABLES}

Page

Table 1. Measured Pressures (Torr) for each enrichment level ........................................12

Table 2. Analysis results for the special background cases.......................................21

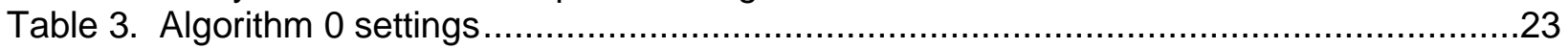

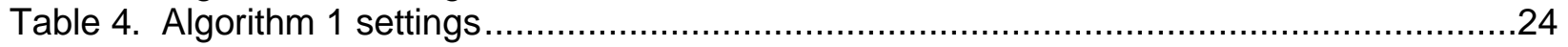

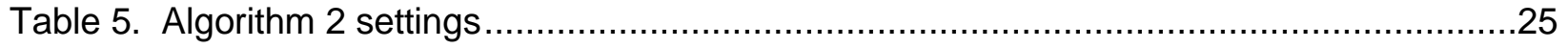

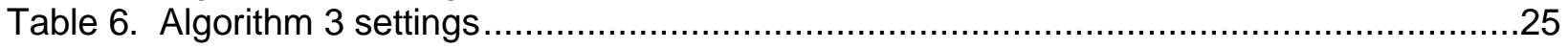

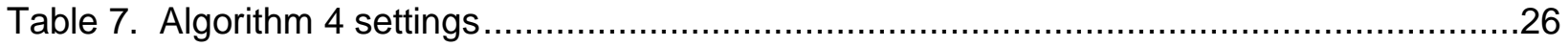

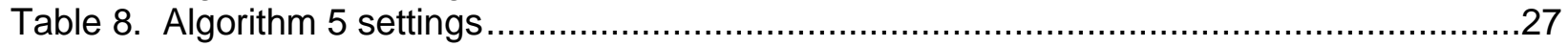

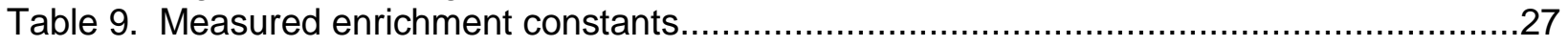

Table 10. Measured ROls for each algorithm, enrichment, and pressure ...........................31 


\section{Revision History}

Rev0 - August 2014

Rev 1 - December 2015

- Recomputed calibration constants for updated ROI regions and algorithms using the data collected in August 2014.

Valid for software version 866 dated 2015/11/09 14:57:58 


\section{$1.0 \quad$ INTRODUCTION}

As global uranium enrichment capacity under international safeguards expands, the International Atomic Energy Agency (IAEA) is challenged to develop effective safeguards approaches at gaseous centrifuge enrichment plants while working within budgetary constraints. The "Model Safeguards Approach for Gas Centrifuge Enrichment Plants" (GCEPs) developed by the IAEA Division of Concepts and Planning in June 2006, defines the three primary Safeguards objectives to be the timely detection of: 1) diversion of significant quantities of natural (NU), depleted (DU) or low-enriched uranium (LEU) from declared plant flow, 2) facility misuse to produce undeclared LEU product from undeclared feed, and 3) facility misuse to produce enrichments higher than the declared maximum, in particular highly enriched uranium (HEU). The IAEA continues to pursue innovative techniques and an integrated suite of safeguards measures to address the verification challenges posed by advanced centrifuge technologies and the growth in separative work unit capacity at modern centrifuge enrichment plants.

The ability to continuously and independently (i.e. with a minimum of information from the facility operator) monitor not only the uranium mass balance but also the ${ }^{235} \mathrm{U}$ mass balance in the facility could help support all three verification objectives described above. Two key capabilities required to achieve an independent and accurate material balance are 1) continuous, unattended monitoring of in-process $\mathrm{UF}_{6}$ and 2) monitoring of cylinders entering and leaving the facility. Descriptions and analysis of instrumentation concepts and potential implementation options to provide these capabilities can be found in "Design, Modeling and Viability Analysis of an Online Uranium Enrichment Monitor" by L. Eric Smith and Alain R. Lebrun, IEE NSS 2011.

The continuous monitoring of in-process $\mathrm{UF}_{6}$ (Figure 1) would rely on a combination of load-cell monitoring of the cylinders at the feed and withdrawal stations, online monitoring of gas enrichment, and a high-accuracy net weight measurement of the cylinder contents. The Online Enrichment Monitor (OLEM) is the instrument that would continuously measure the timedependent relative uranium enrichment, $E(t)$, in weight percent ${ }^{235} \mathrm{U}$, of the gas filling or being withdrawn from the cylinders.

The primary purpose of previous online enrichment monitors deployed by the IAEA, called the Continuous Enrichment Monitor (CEMO), was "Go/No-Go" detection of highly enriched uranium production, and these instruments were located on the low-pressure side of the header pump. The OLEM, however, will be located on the high-pressure portion of the unit header pipe (Figure 1), a location that is advantageous for quantitative assay because of the relatively strong gas signal and the opportunity to directly monitor the gas pressure (fewer proprietary concerns from operators, compared to the low-pressure portions of the plant). Other measurement and analysis challenges arise at this location, however, such as gas-pressure transients when cylinders are attached and removed from withdrawal stations.

The OLEM design concept (Figure 1) combines gamma-ray spectrometry using a collimated $\mathrm{Nal}(\mathrm{TI})$ detector with gas pressure and temperature data to calculate the enrichment of the $\mathrm{UF}_{6}$ gas within the unit header pipe as a function of time. The $\mathrm{UF}_{6}$ gas pressure can be measured using a dedicated IAEA pressure sensor or a non-invasive receiver/transmitter device installed on one of the operator's pressure gauges. Temperature of the $U F_{6}$ gas inside the pipe is inferred using temperatures sensors (e.g., resistance temperature detectors) attached to the outside of the header pipe and thermally insulated from the room environment.

The OLEM described in this document addresses only the relative enrichment measurement portion of the GCEP safeguards concepts being explored by the IAEA. Integration of the OLEM data streams with other data streams in a suite of facility-level safeguards measures (e.g. load 
cell data, accountancy scale data, and cylinder verification data) is not a part of the development effort described here. Concepts and approaches for such integration continue to be explored by the IAEA, as technology options develop and are better understood. Ultimately, technical measures will be implemented on a facility-by-facility basis, based on negotiations with state authorities.

The OLEM system architecture will include a network of Collection Nodes. Multiple Collection Nodes will be supported by a Junction Box, for communication and backup power. An IAEA data monitoring station ("IAEA Cabinet") will provide a user console for monitoring of OLEM Collection Nodes, and will serve as an integration point for other safeguards instrumentation at the facility (e.g. operator load cells and cylinder verification stations).

Figure 1 shows the OLEM Collection Node (blue boxes), which combines gamma-ray spectrometry using a $\mathrm{Nal}(\mathrm{TI})$ detector with temperature and gas pressure data to calculate the relative enrichment of the gas as a function of time, $\mathrm{E}(\mathrm{t})$. The location of the Continuous Enrichment Monitor (CEMO), IAEA's previous generation of online enrichment monitors, intended only for simple Go/No-Go detection of HEU production within an individual cascade, is also shown.

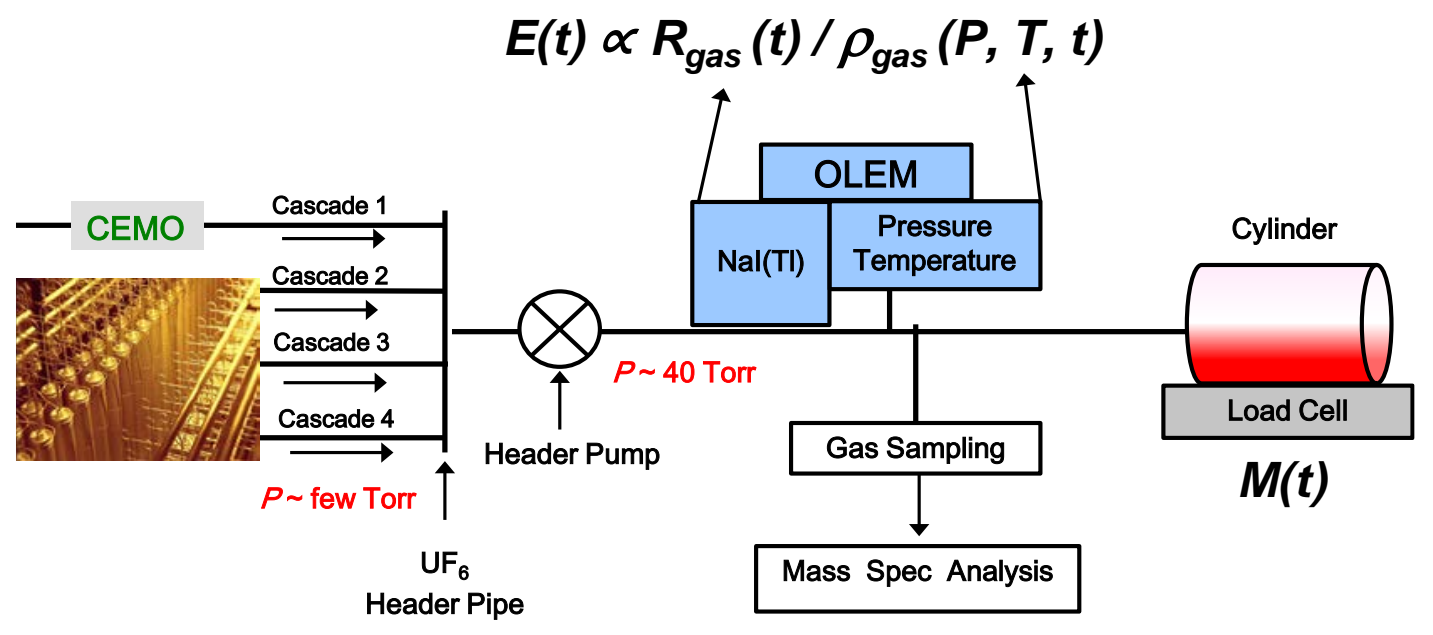

Figure 1. OLEM Collection Node 


\subsection{SUMMARY}

The OLEM components have been tested on the ORNL UF 6 flow loop. Data has been collected at five different enrichment levels $(0.71 \%, 2.97 \%, 4.62 \%, 6.0 \%$, and $93.7 \%)$ at several pressure conditions. The test data has been collected in the standard OLEM N.4242 file format for each of the conditions with a 10-minute sampling period and then averaged over the span of constant pressures.

Analysis of the collected data has provided enrichment constants that can be used for 1.5" stainless steel schedule 40 pipe measurement sites. The enrichment constant is consistent among all the wide range of enrichment levels and pressures used.

The current Revision 1 of this report is a reanalysis of the data collected in August 2014 with the latest software algorithms (version 866 dated 2015/11/09 14:57:58). The ROI ranges have also been refined to optimize the results of various algorithms. The ROI ranges are somewhat arbitrary since a large number of solutions exist that provide acceptable results. The current implementation of OLEM algorithms use two suggested ROI ranges: a "small ROI", which is set between 165 and $220 \mathrm{keV}$, and "large" ROI, which is set between 120 and $220 \mathrm{keV}$. The large $\mathrm{ROI}$, includes the $143 \mathrm{keV}$ peak, and is used when maximizing the number of counts is of interest. The small ROI is set exclusively around the $186 \mathrm{keV}$ peak and is used in applications when minimizing the room background is the primary concern. Other ROI ranges may be used with OLEM, but the calibration constants in this report would not apply.

\subsection{EXPERIMENTAL SETUP}

These tests utilized the $\mathrm{UF}_{6}$ Test Loop, a laboratory-scale apparatus at ORNL built and utilized for the purpose of providing a $U_{6}$ environment for testing of instruments and materials in simulated operating plant conditions.

\subsection{ORNL UF 6 LOOP}

The laboratory system utilized consists of a gas handling manifold (test loop) constructed of fluorine-compatible materials (largely Monel, nickel, and SS316). It contains a gas circulation loop driven by a metal bellows pump to produce the desired gas flow and to ensure mixing of gases (important for assuring that the on-line gas analyses utilize a representative sample during long experimental runs). On-line instrumentation includes a mass flowmeter, several pressure and temperature sensors and a Fourier transform infrared (FTIR) spectrophotometer.

The FTIR is capable of determining partial pressures for most of the gaseous compounds of interest. In this project, these were $\mathrm{UF}_{6}$ and its principal gaseous reaction product with water, HF (hydrofluoric acid). Spectra can be displayed in real time and are also recorded for later detailed analysis. Other environmental data (temperature, flow and total pressure) are monitored and recorded using a data acquisition (DAQ) system. Both the FTIR and the DAQ can be configured to take readings at fixed intervals, allowing unattended monitoring of the system for extended periods of time, i.e., overnight or through a weekend.

Auxiliary systems include a dry vacuum pumping system protected by a soda-lime / activated alumina chemical trap, gas sources $\left(\mathrm{He}, \mathrm{N}_{2}\right.$, and $\mathrm{F}_{2}$ ), and sampling systems. Figure 2 shows a schematic of the apparatus in the configuration used for this test showing the 1.5" Schedule 40 Stainless Steel pipe section.

The test loop is located in a radiological and chemical fume hood, while the instrumentation controls and readouts, as well as the DAQ computers and FTIR, are located adjacent to the 
hood. Operational safety and radiological constraints vary with the gases used, the assay and quantity of uranium used, operations to be conducted, etc. This project, being very nonintrusive, experienced no difficulty in putting the detectors into the hood and recovering them afterward free of radioactive contamination.

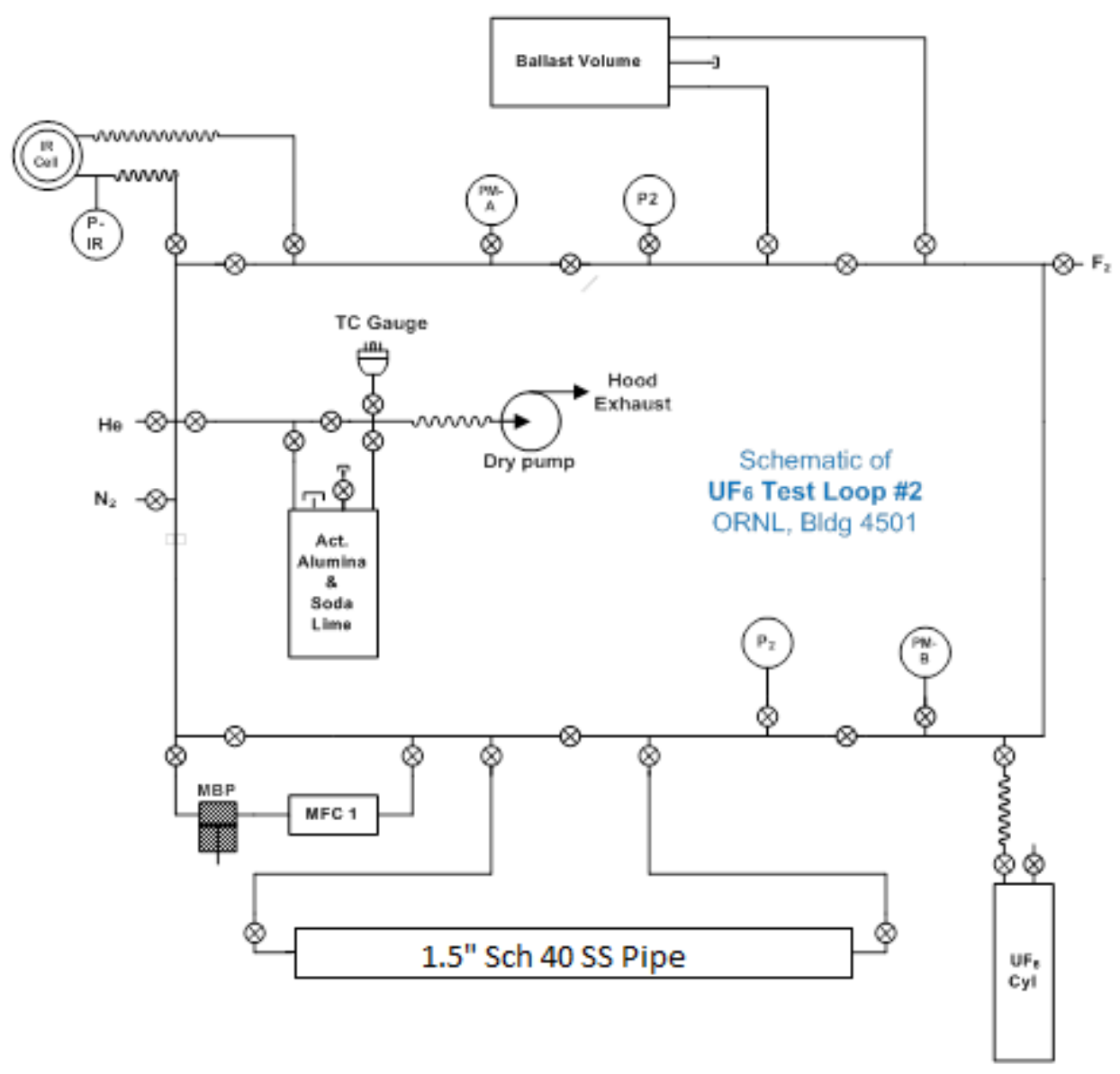

Figure 2. Schematic of $\mathrm{UF}_{6}$ Test Loop \#2 as configured for this testing

From a gas handling standpoint, the experimental process was straightforward. The Test Loop was initially pumped down to as low a vacuum as was readily achievable (on the order of 0.1 Torr), then isolated. $\mathrm{UF}_{6}$ of the desired assay was then desublimed from the source cylinder into the system up to the desired pressure. An FTIR scan was taken and analyzed to assure that the gas was predominantly $\mathrm{UF}_{6}$ (the head space of $\mathrm{UF}_{6}$ cylinders can contain residues of non-condensable gases, so it is advisable to determine the $\mathrm{UF}_{6}$ partial pressure by other than a simple pressure gauge measurement). The gas, static to this point, was then circulated and, typically, the FTIR was put into automatic scan mode, taking and recording spectra at preset intervals (typically every half hour or hour).

When it was desired to raise the $\mathrm{UF}_{6}$ partial pressure for a later phase of the experimental campaign, more $\mathrm{UF}_{6}$ was added from the source cylinder. If it was desired to reduce the partial pressure, the desired amount was reduced either by evacuating to the chemical trap (for natural or depleted assays) or by cold trapping (for enriched uranium, which is in shorter supply). 
Selected FTIR spectra were analyzed manually as needed during the experimental run, and afterward, all the accumulated spectra were analyzed via an automated off-line system. With the spectra plotted in Absorbance Units, each characteristic absorbance band of a particular compound is proportional to the partial pressure of that compound in the $10 \mathrm{~cm}$ IR cell

(practically speaking, within limits dictated by the instrument baseline noise level at the low end and peak saturation at the high end). For $U_{6}$, well established absorbance-to-partial pressure factors were used for several peaks to determine the $U_{6}$ partial pressure. HF bands are subject to a phenomenon known as pressure broadening which essentially renders the peak height non-linear with respect to pressure. A correction was made to account for pressure broadening in HF.

In these experiments, it was observed that the $\mathrm{UF}_{6}$ partial pressure slowly declined during an otherwise undisturbed experiment and the HF partial pressure rose correspondingly. This suggests a slow, continuing source of reaction with, most likely, either water or a hydrate species. Where the water or hydrate came from is unknown. It cannot be attributed to a leak from atmosphere, as a leak of the magnitude necessary to produce the observed HF partial pressure rise would have materially raised the pressure due to the accompanying nitrogen and oxygen, and no such pressure change was observed. That leaves open the possibilities of slow reaction with residual adsorbed moisture or hydrates, or permeation of $\mathrm{H}_{2} \mathrm{O}$ or $\mathrm{HF}$ through elastomer gasket material - the only source of which in this system is the gasket for the Metal Bellows Pump. In any case, the loss of $\mathrm{UF}_{6}$ was not large enough to materially affect the measurements. 


\subsection{OLEM SETUP}

Figure 3 through Figure 8 show the experimental setup for these measurements. The following settings were used for the Osprey MCA:

Voltage

Coarse Gain

Fine Gain

\section{$650 \mathrm{~V}$}

2

2

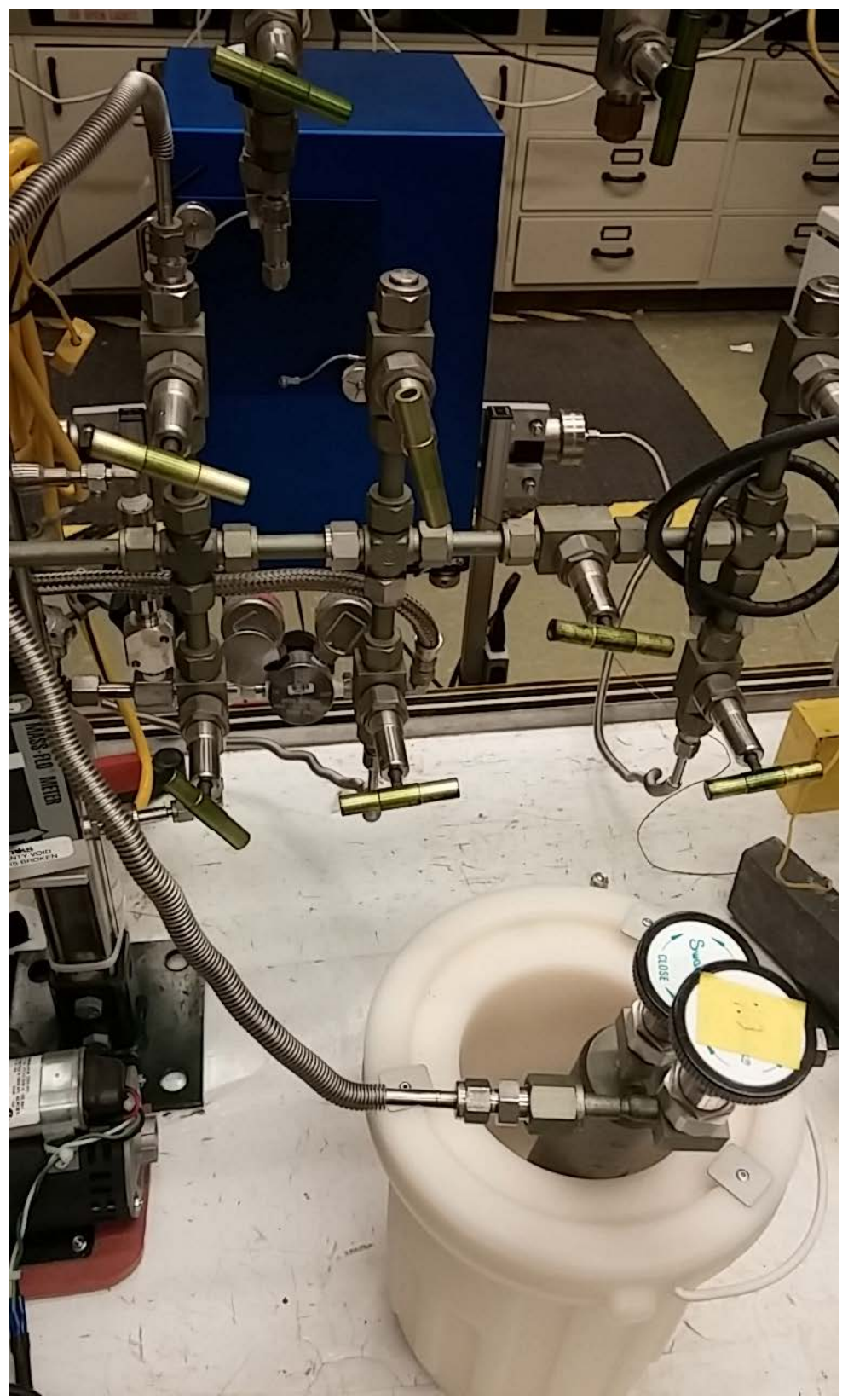


Figure 3. Experimental setup

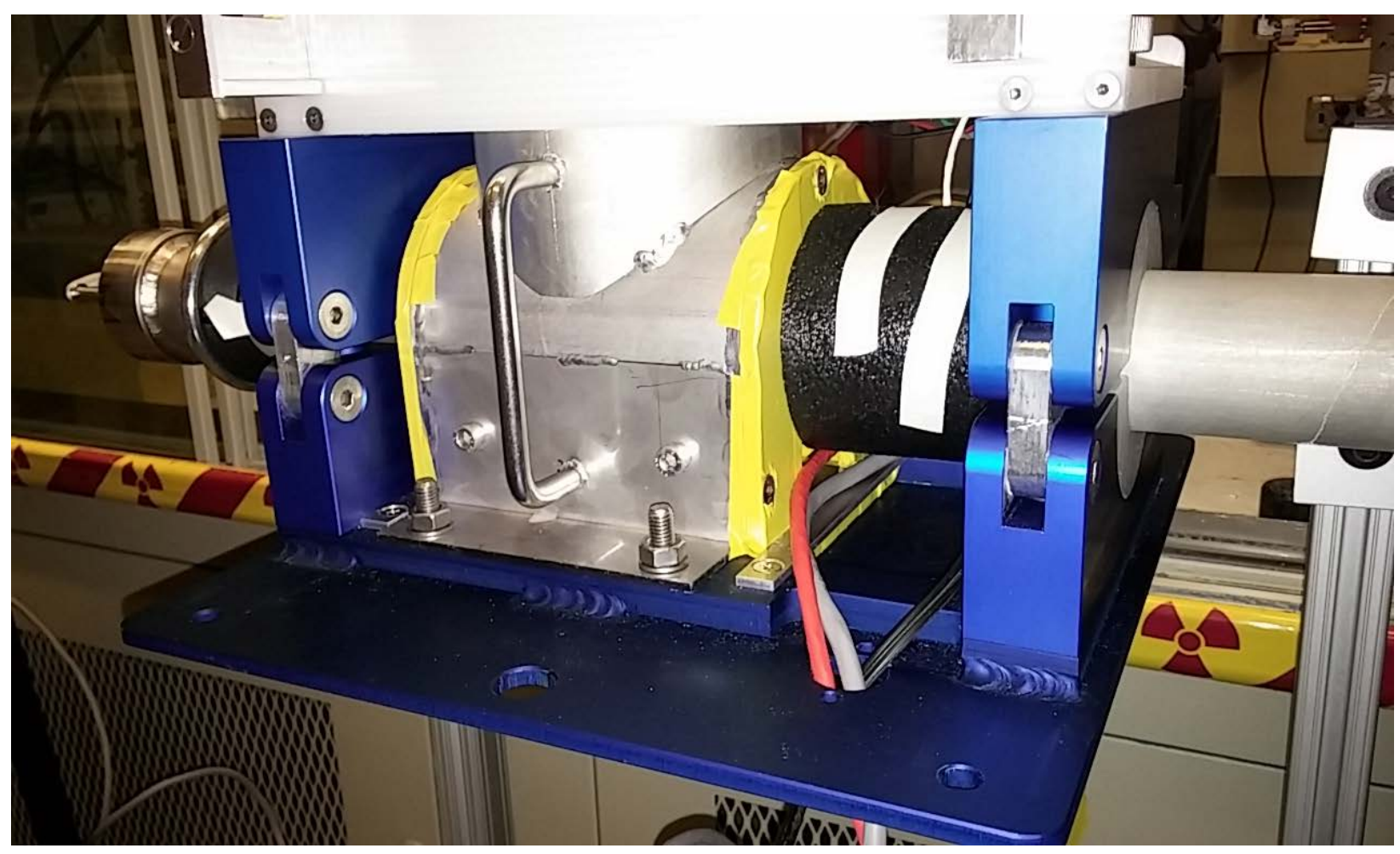

Figure 4. Detail of OLEM detector and shielding on 1.5" pipe 


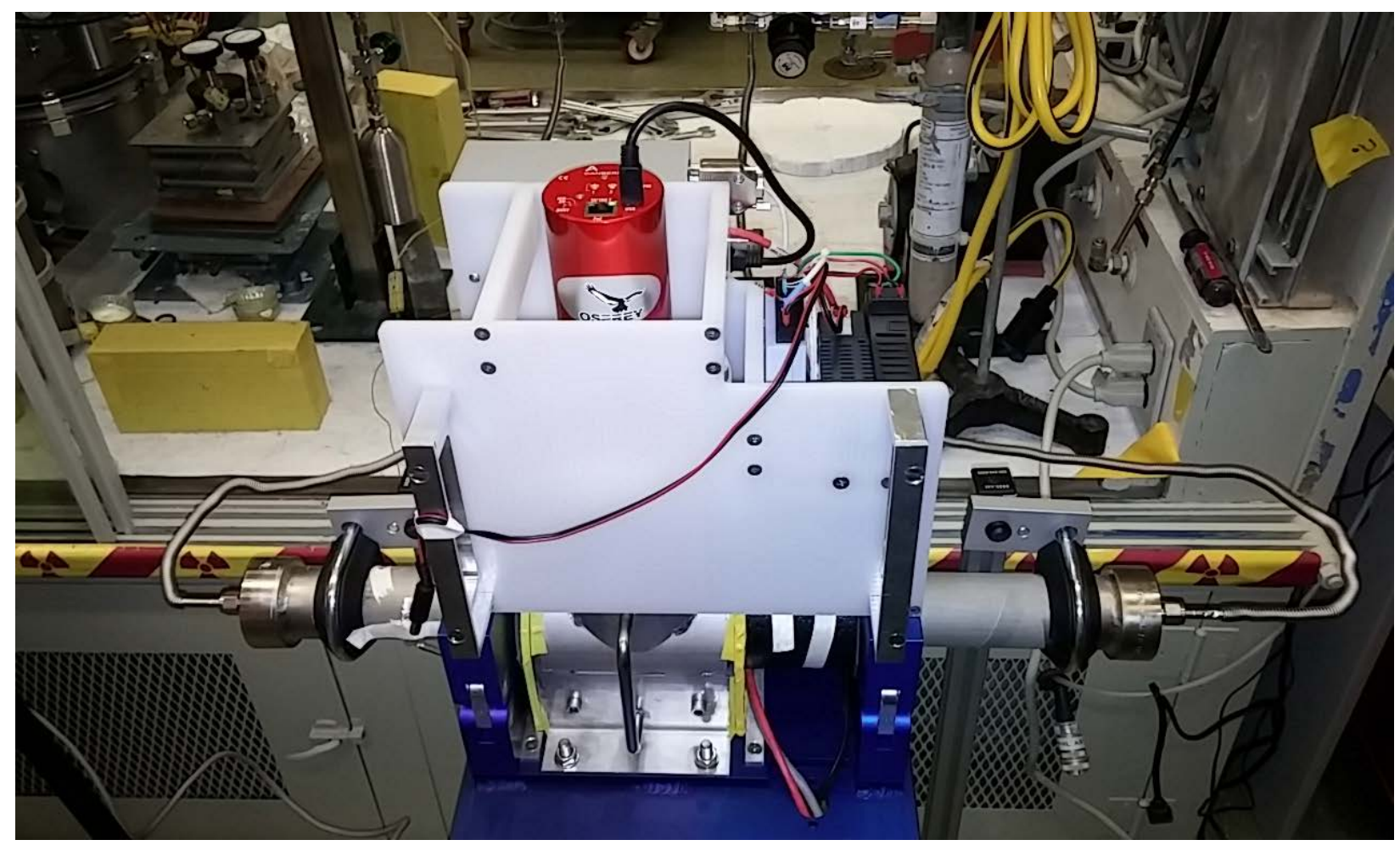

Figure 5. Detail of location of the OLEM 


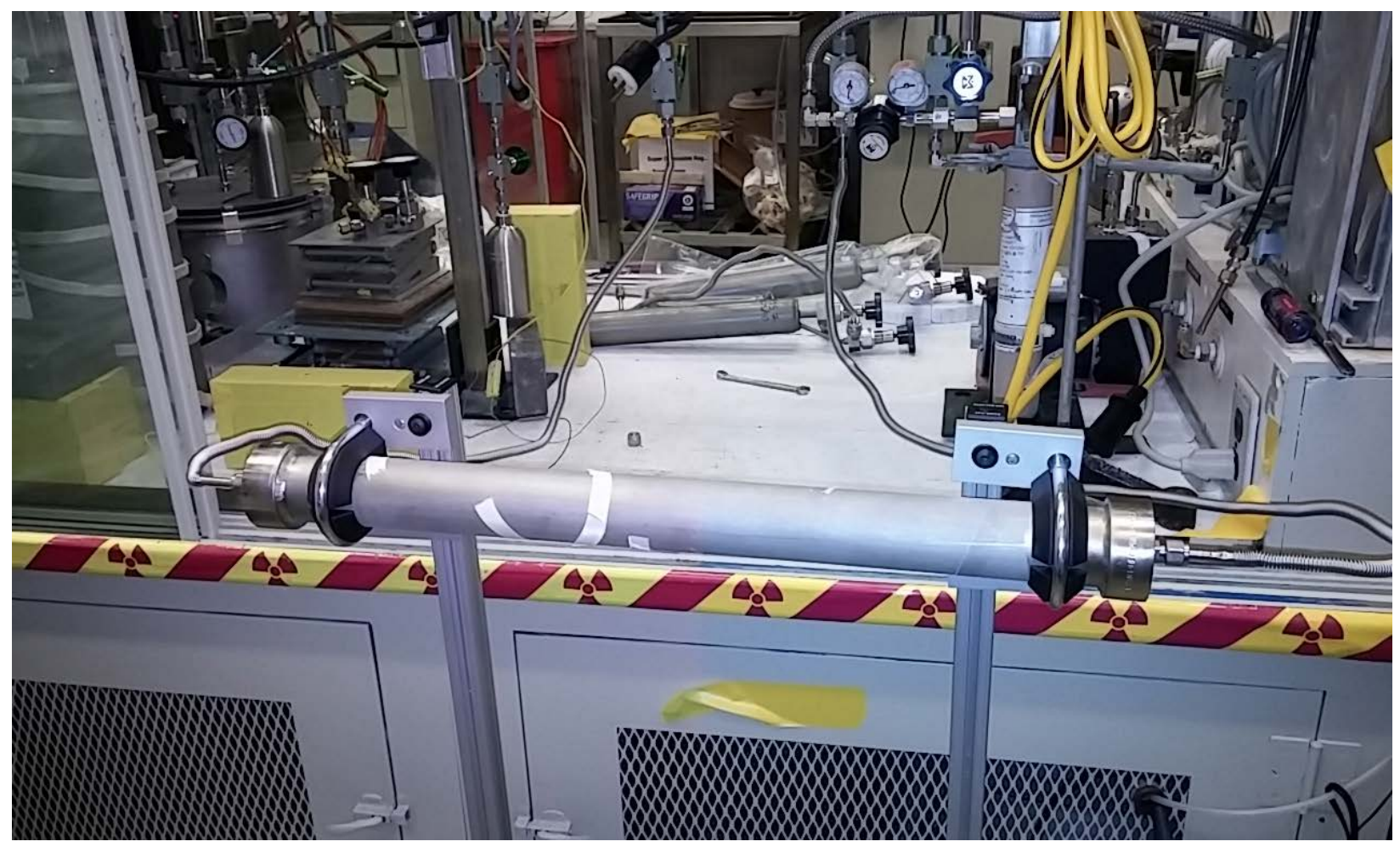

Figure 6. Test loop showing the 1.5" Sch40 SS pipe

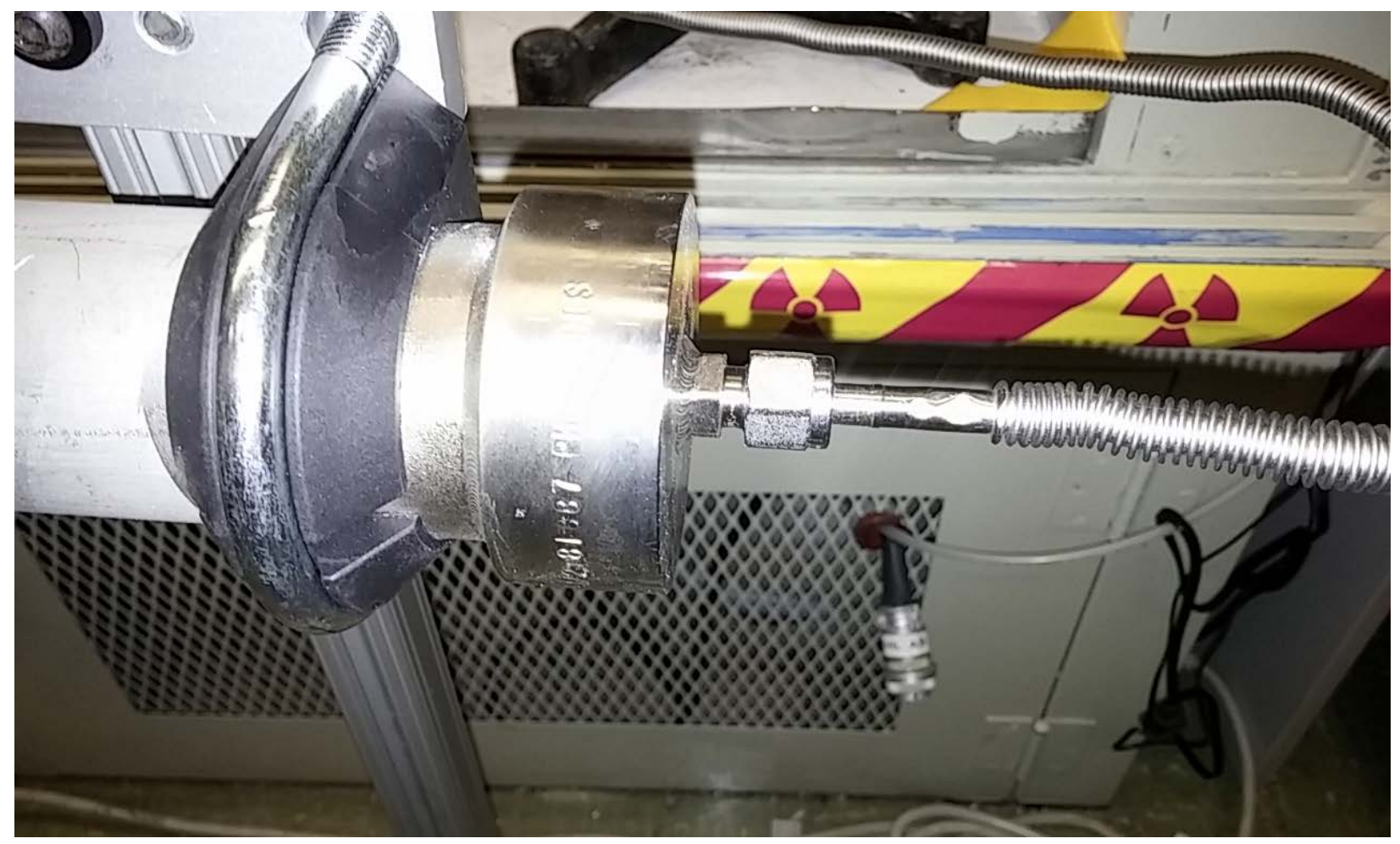

Figure 7. Detail of the pipe connections 


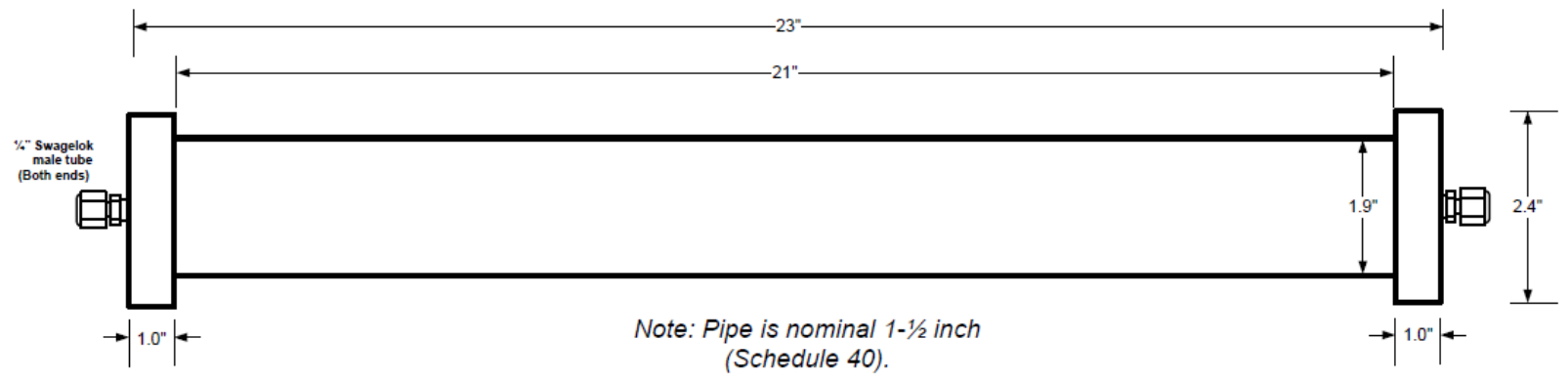

Figure 8. Test section details (not to scale) 


\subsection{MEASURED DATA}

Five $\mathrm{UF}_{6}$ enrichment levels were used for these measurements: $0.71 \%, 2.97 \%, 4.62 \%, 6.0 \%$, and $93.7 \%$. Table 1 shows the pressure levels measured at each enrichment level.

Table 1. Measured Pressures (Torr) for each enrichment level

\begin{tabular}{|r|r|r|r|r|}
\hline $0.71 \%$ & $2.97 \%$ & $4.62 \%$ & $6.00 \%$ & $93.70 \%$ \\
\hline 9.5 & 7.0 & 15.1 & 9.3 & 6.3 \\
\hline 21.3 & 25.2 & 18.0 & 29.0 & 11.2 \\
\hline 35.4 & 42.5 & 25.4 & 57.0 & 14.9 \\
\hline 48.5 & & 30.9 & & \\
\hline & & 35.6 & & \\
\hline & & 41.2 & & \\
\hline & & 45.6 & & \\
\hline
\end{tabular}

The measurements were conducted over a two-week period with step changes in pressure accomplished by either allowing additional product in the loop or by removing it via liquid nitrogen cooling or simply discarding to a chemical trap. The tests were run from August 4 through August 15, 2014.

As expected, a number of unexpected mishaps occurred during testing. Only "good data" was used for the analyses, and unreliable data was discarded. The loop was passivated with $\mathrm{F}_{2}$ prior to the start of tests, and the stainless pipe was heated to $80 \mathrm{C}$ for degassing. It was also passivated in between the different enrichment runs.

Figure 9 through Figure 13 show the measured spectra.

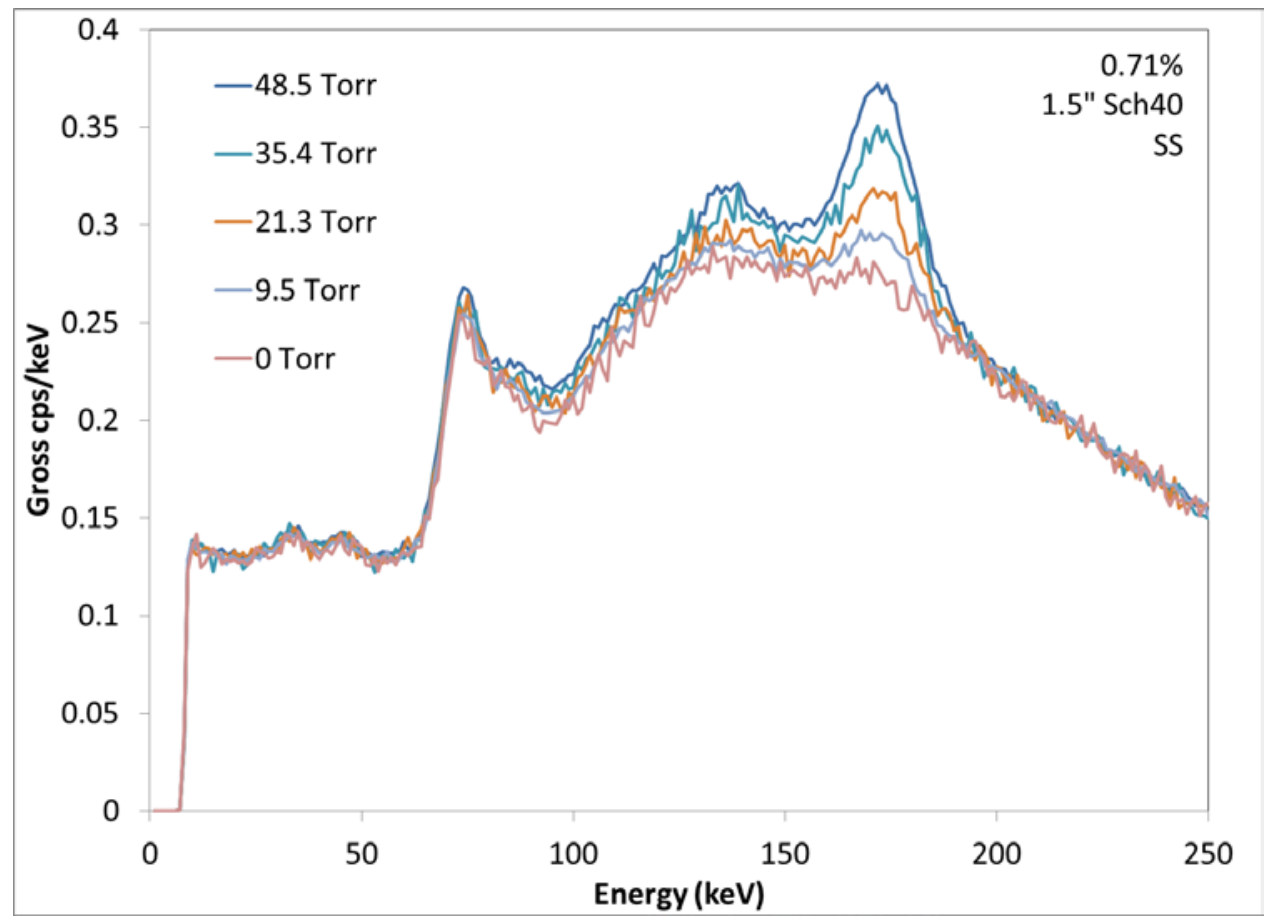

Figure 9. Average spectra at different pressures for $0.71 \%$ data 


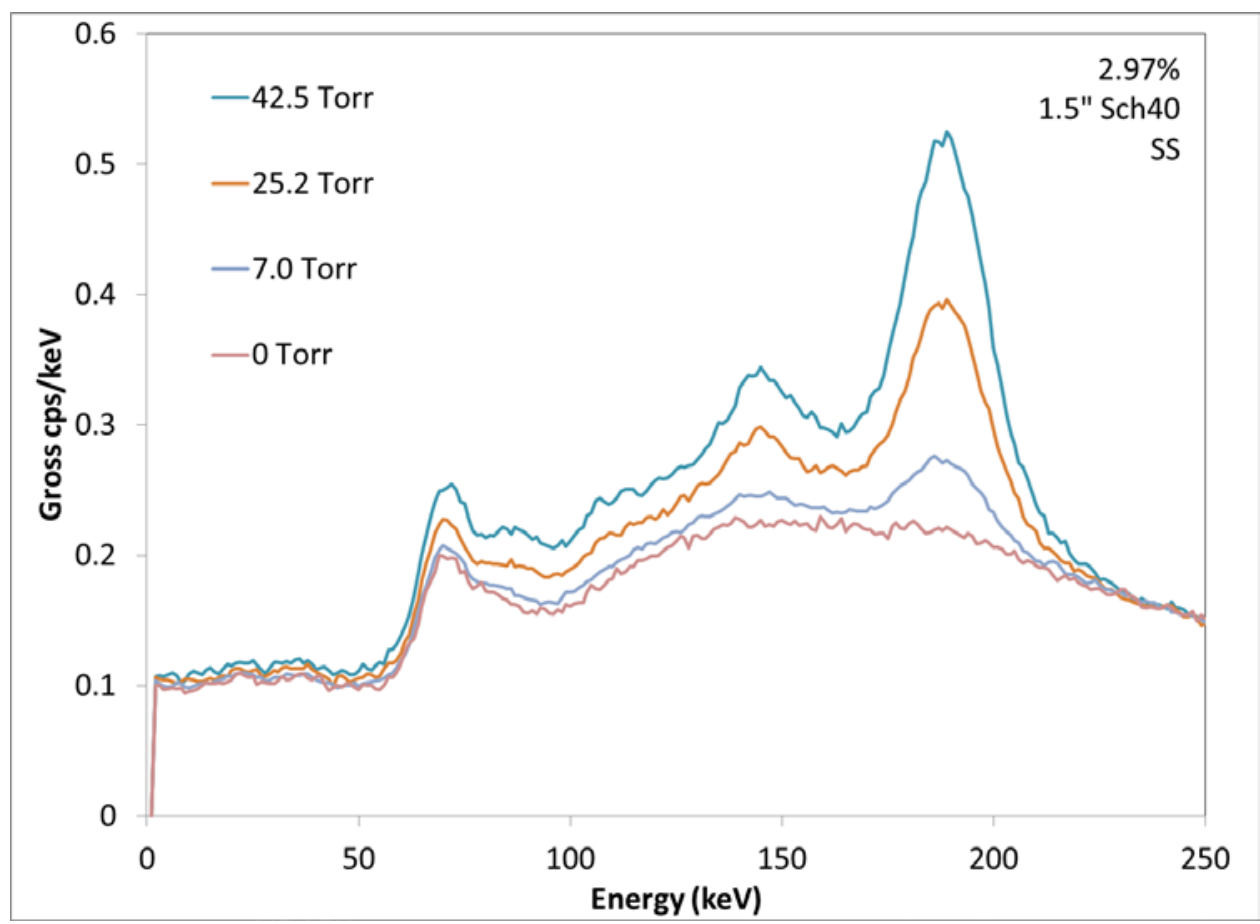

Figure 10. Average spectra at different pressures for $2.97 \%$ data

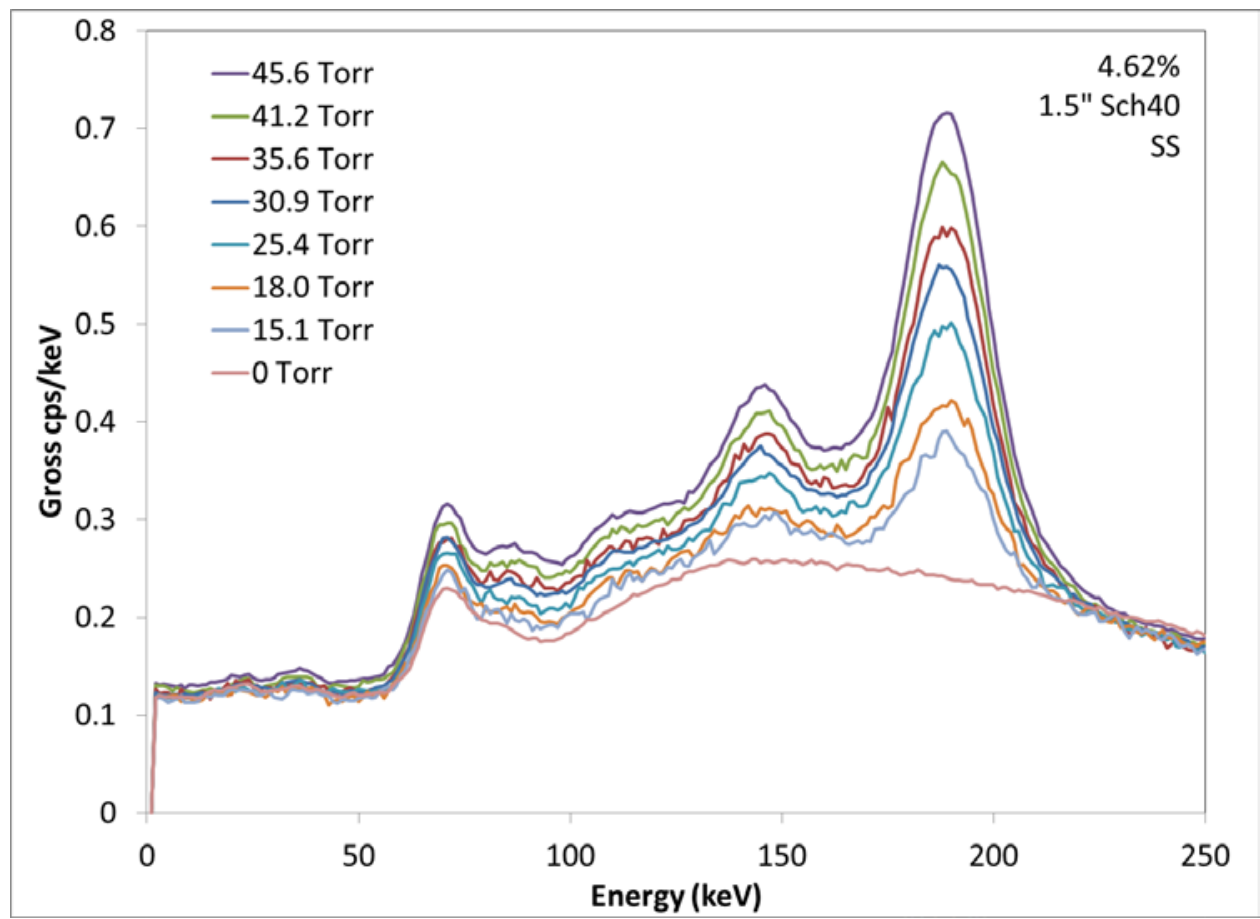

Figure 11. Average spectra at different pressures for $4.62 \%$ data 


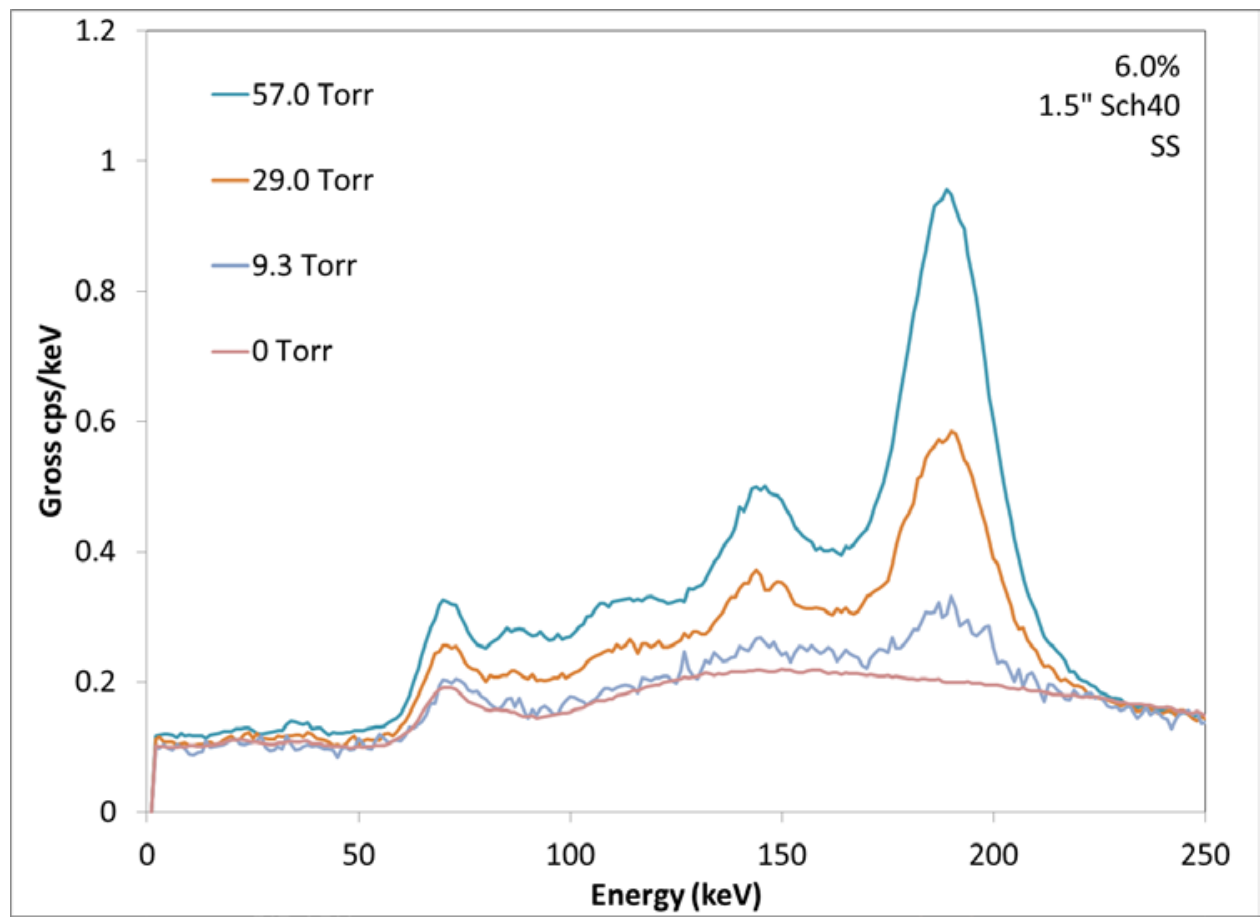

Figure 12. Average spectra at different pressures for $6.0 \%$ data

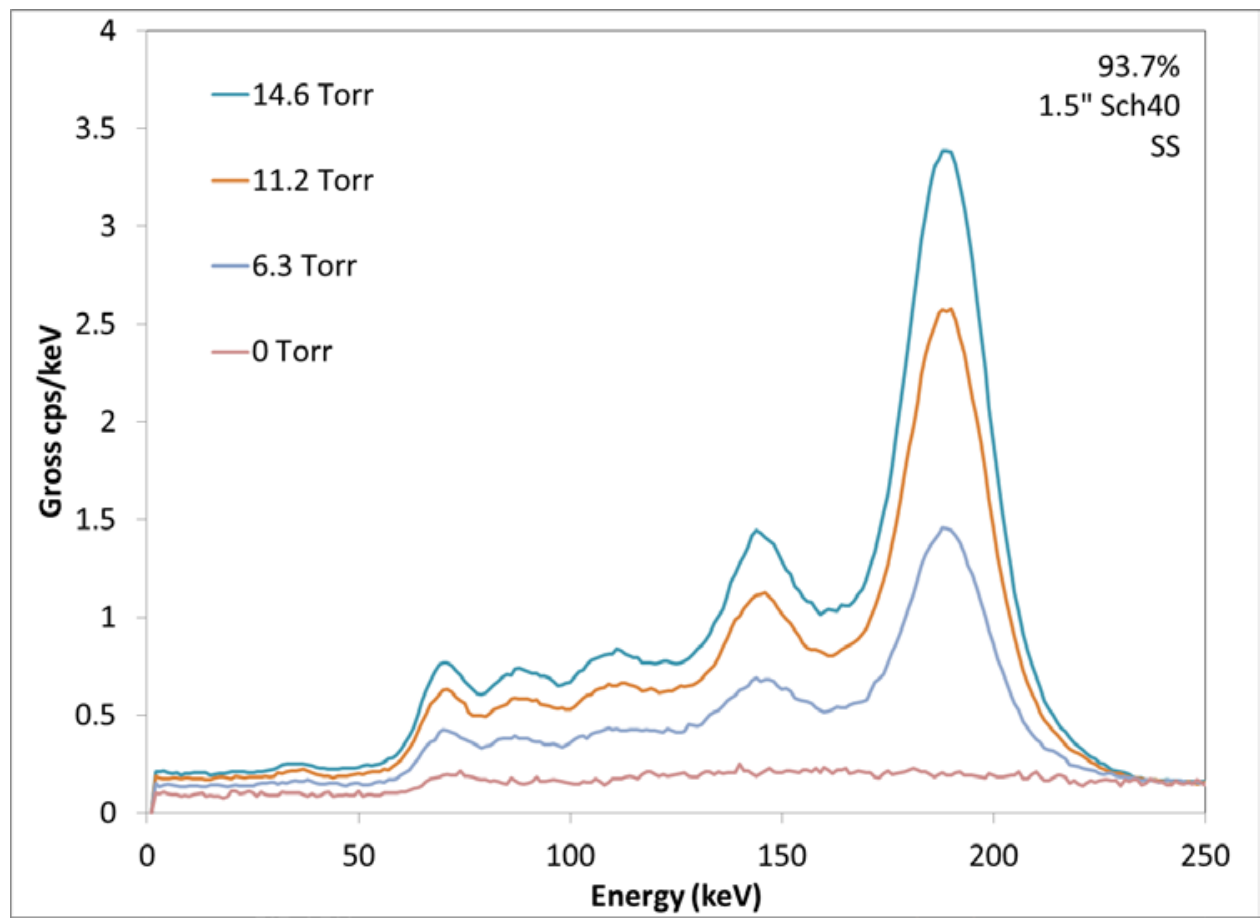

Figure 13. Average spectra at different pressures for $93.7 \%$ data 


\subsection{OLEM METHODOLOGY}

The OLEM methodology processes the measured spectra as function of energy to convert it to a single number, which we refer to as the "186 count-rate," even though a more appropriate description would be the "count rate that is proportional to ${ }^{235} \mathrm{U}$ density." Spectra are measured as function of time. Typically, a spectrum was obtained every 10 minutes, but the interval is user-adjustable. The average pressure and temperature during the 10-min interval is also recorded by measuring these variables with a fine time step (typically every $\sim 10$ seconds). Data is collected for a sufficient period of time (i.e., several 10-min blocks are collected), and the count-rate as function of time is analyzed. A linear regression is performed on the data; the zero intercept is the background (signal at zero pressure), and the slope is directly proportional to the enrichment. The concept is illustrated in Figure 14 using computer generated data.

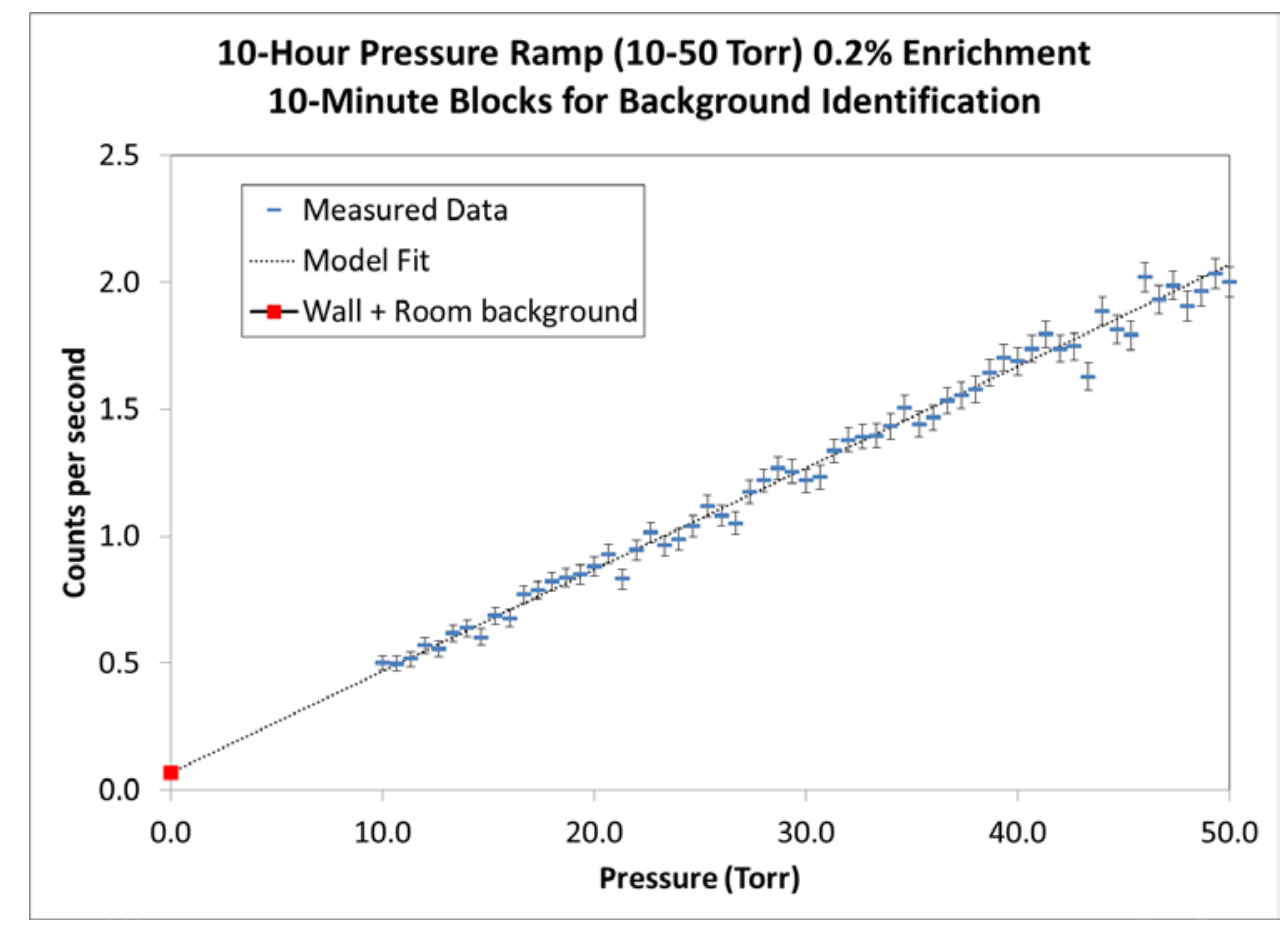

Figure 14. Illustration of OLEM algorithms application to simulated data

Five algorithms have been programed in OLEM. They are labeled Alg00 -Alg05. The primary difference between the algorithms is the methodology used to convert the energy-dependent spectra to a single net count-rate number.

\subsection{ALG00}

Alg00 is the simplest algorithm. It uses the standard region of interest (ROI) approach, where anchor points are set around the $186 \mathrm{keV}$ region of interest. A trapezoidal region is defined below the anchor points; the area of this region is assumed to be the uncorrelated background, or background under the peak. The area above is assumed to be the 186 count-rate. This method is a very common method of background reduction used in the Non-Destructive Analysis (NDA) community, and it is illustrated graphically in Figure 15. 
For Alg00, the 186 count rate is obtained by removing the trapezoidal background as shown in Figure 15, and then the process of Figure 14 is used to identify the remaining background, if any which is within the peak, for example from wall deposits.

Alg00 is expected to have the worst statistics, because the trapezoidal background removal depends on the statistics of the anchor points, which have low counts (bottom of the peak) and small energy width. For these analyses, we used $5 \mathrm{keV}$ for the anchor points.

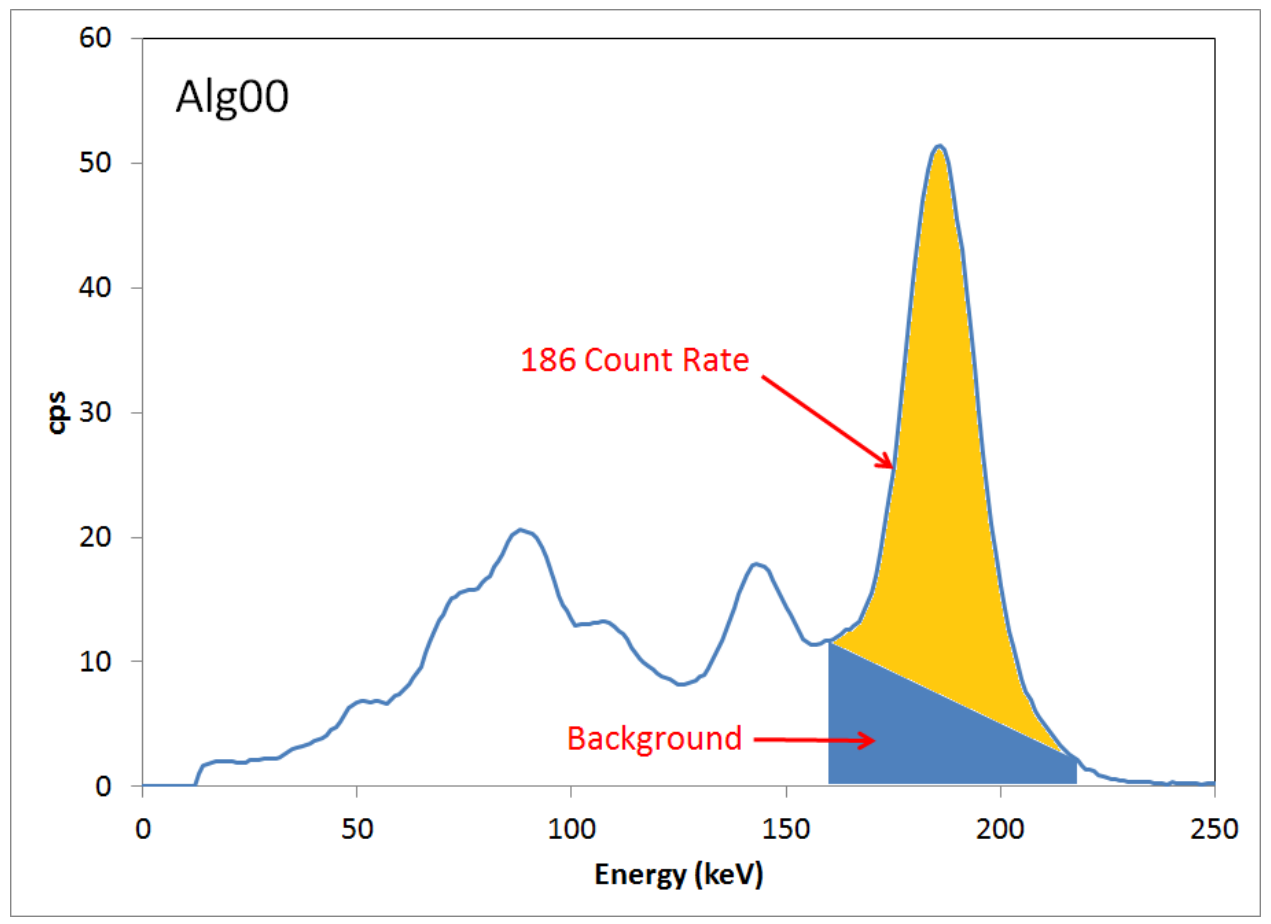

Figure 15. Illustration of Alg00 trapezoidal background removal under the peak. 


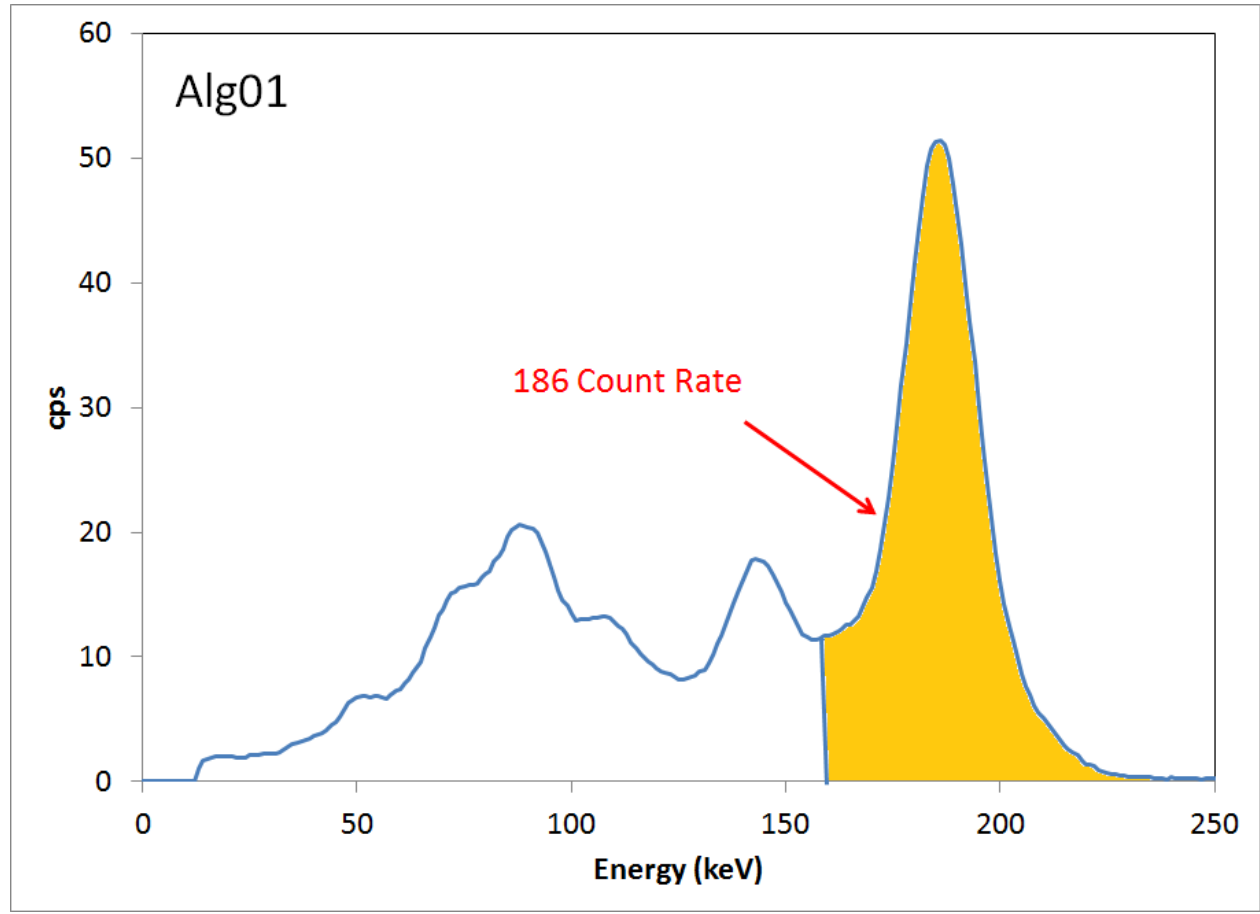

Figure 16. Illustration of Alg01 count rate methodology

\subsection{ALG01}

Since the OLEM algorithm automatically differentiates the background from the signal as illustrated in Figure 14, there is no need to attempt to remove the room background (background under the peak) by the Alg00 trapezoidal removal methods. Alg01 takes advantage of this fact and simply defines the "186 count rate" as an ROI count around the peak, as illustrated in Figure 16. This algorithm has the advantage of not adding additional statistical fluctuations caused by the selection of anchor points. In addition, the count rate itself is slightly larger than Alg00 and has better statistics, although the total statistical uncertainty would be similar.

\subsection{ALG02}

Alg02 is similar to Alg01. But because we are interested in the part of the signal that is proportional to the ${ }^{235} \mathrm{U}$ density, Alg02 includes the $143 \mathrm{keV}$ peak, which is also emitted by ${ }^{235} \mathrm{U}$. Alg02 is illustrated in Figure 17. This larger ROI provides for additional counts and better counting statistics for most situations. A disadvantage of Alg02 is that the background counts increase over Alg01 because the ROI is larger; thus, it is not always guaranteed that Alg02 will have better counting statistics than Alg01, even though experience has shown that Alg02 tends to outperform Alg01 in almost all cases studied. 


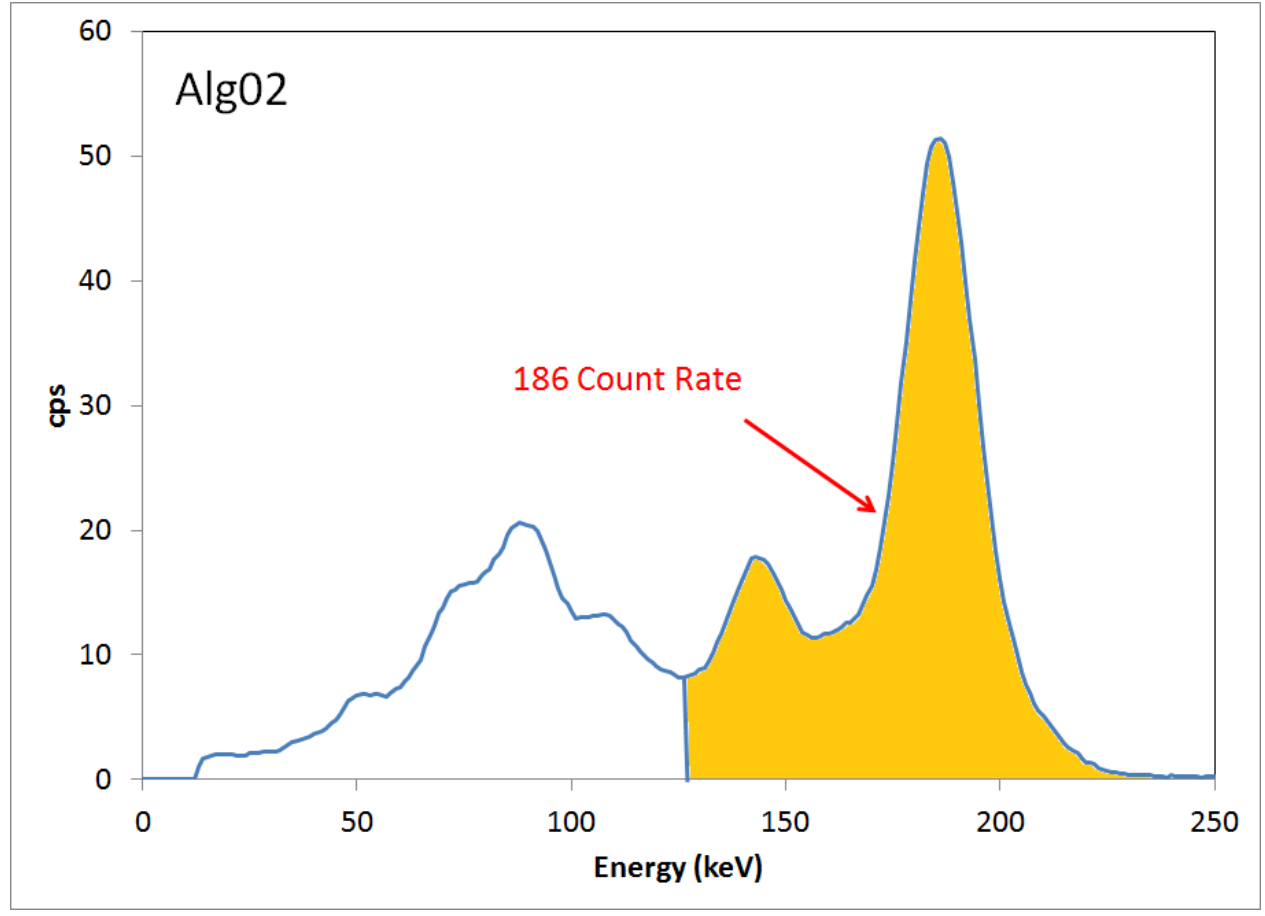

Figure 17. Illustration of Alg02 count rate methodology

By looking at Figure 17, an obvious algorithm extension would be to include the complete energy region (for example, from $10 \mathrm{keV}$ to $250 \mathrm{keV}$ ). However, the peaks around $100 \mathrm{keV}$ are $\mathrm{X}$-Ray peaks. X-Rays are the result of inner shell electrons being excited out of orbit by an external perturbation. In the case of Uranium, the naturally occurring 186 and $143 \mathrm{keV}$ gamma rays are the excitation source. For this reason, Uranium X-Rays are proportional to the product of the ${ }^{235} U$ density times the density of total Uranium. Because of this dependency on the density square and also geometry, X-Rays cannot be used to define the ${ }^{235} U$ density with the extreme high accuracy required by the OLEM application (though they may be used if the accuracy requirements were relaxed).

\subsection{ALG03}

Alg03 is reserved for the LANL implementation.

\subsection{ALG04}

Algorithm Alg04 is similar to Alg02, and it uses the same ROI as in Figure 17. The difference between these two algorithms is that Alg04 utilizes a user-supplied background, for both the background under and within the peak, and it does not attempt to identify it adaptively.

Alg04 is useful for applications where the pressure is constant or not expected to change significantly. For these applications, the enrichment constant must be known from a series of calibration runs with the same exact geometry. Then, using a single-pressure measurement, the background (counts at zero pressure) can be obtained manually by extrapolating the measured counts with the known enrichment constant and enrichment.

The enrichment is obtained from the following equation:

$$
\varepsilon=K(R-B) / \rho
$$


where $\varepsilon$ is the enrichment, $\mathrm{K}$ is the enrichment constant, $\mathrm{R}$ is the ROI gross counts, $\mathrm{B}$ is the background, and $\rho$ is the gas density (a function of pressure and temperature).

With a single pressure-level measurement, known enrichment constant and a declared enrichment, the equation can be rearranged and the background estimated as

$$
B=R-\varepsilon \rho / K
$$

The latest version of the software algorithms includes the option of removing the background contribution in the ROI of interest from Compton scattering of high-energy gamma rays (e.g., $\mathrm{Pa}$-234). To this end, the OLEM algorithm calculates a variable (ROI_bckg), which is the total $\mathrm{ROI}$ counts between 250 and $400 \mathrm{keV}$. The configuration file input field <bckg_ROI> provides the user with the ability to input two parameters $\left(B_{0}\right.$ and $\left.B_{1}\right)$, so that the background is computed as

$$
\mathrm{B}=\mathrm{B}_{0}+\mathrm{B}_{1}{ }^{*} \mathrm{RO} \_ \text {bckg }
$$

This form for the background has been proven with experimental data to remove most of the variable room background contributions with acceptable accuracy.

\subsection{ALG05}

Alg05 is similar to Alg00 and Figure 15. It defines a trapezoidal background under the peak, but the anchor points of the trapeze are evaluated adaptively by the algorithm, which tries to adjust a best fit to the theoretical U-235 spectrum. .

The basic idea of Alg05 is that the spectrum around the $186 \mathrm{keV}$ peak can be represented by two components:

A set of 5 Gaussian peaks that represent the $U 235$ spectrum, and

A trapeze-shaped area that represents the room background (see Figure 15)

Thus the spectrum, $S(E)$, has the form

$$
S(E)=T(E)+G(E)
$$

Where $T(E)$ is the trapezoidal part and $G(E)$ is the ${ }^{235} U$ Gaussian part.

$T(E)$ is expressed mathematically as

$$
T(E)=a_{0}+\left(a_{1}-a_{0}\right)\left(E-E_{0}\right) /\left(E_{1}-E_{0}\right)
$$

And $G(E)$ is based on the theoretical $235 \mathrm{U}$ spectrum as

$$
\begin{aligned}
& G(E)=A \Sigma\left[G_{i}(E)\right] \quad(i=1,5) \\
& \text { Where } G_{i}(E)=A_{i} \exp \left(-\left(E-E_{i}\right)^{2} / 2 \sigma_{i}^{2}\right) / 2.506 \sigma_{i}
\end{aligned}
$$

$E_{i}$ are the ${ }^{235} U$ peak energies:

$$
\text { 143.76, 163.33, 185.72, 202.11, and } 205.31 \mathrm{keV}
$$

$\sigma_{i}$ are related to the peak full width half $\max (F W H M)$ values, which are scaled by SQRT(E). Initial guess values are:

\section{8, 7.4, 8.3, 8.6, and 8.8}

$A_{i}$ are the relative peak yield values, based on the theoretical ${ }^{235} U$ spectrum:

$10.96,5.08,57.2,1.08$, and 5.01 
Alg05 applies a minimum least squares method to the measured spectrum and obtains the adjustable algorithm parameters that best fit the data. The algorithm identifies the total peak amplitude, $A$, the channel where the 186 peak is located (the other $E_{i}$ values are adjusted relative to this one), and the 186 peak FWHM (the other $\sigma_{i}$ values are adjusted relative to this one). It also identifies the anchor points of the trapeze $\left(a_{0} \& a_{1}\right)$. The final output is the total number of counts under the $186 \mathrm{keV}$ peak Gaussian, which is the difference between the green and red lines in Figure 18. Note that the red line (background) in Figure 18 contains the contributions from the trapeze plus the remaining four ${ }^{235} \mathrm{U}$ peaks $(143.76,163.33,202.11$, and $205.31 \mathrm{keV})$.

As with Alg00, the Alg05 186 net count rate is obtained by subtracting the user supplied background (within the peak) value.

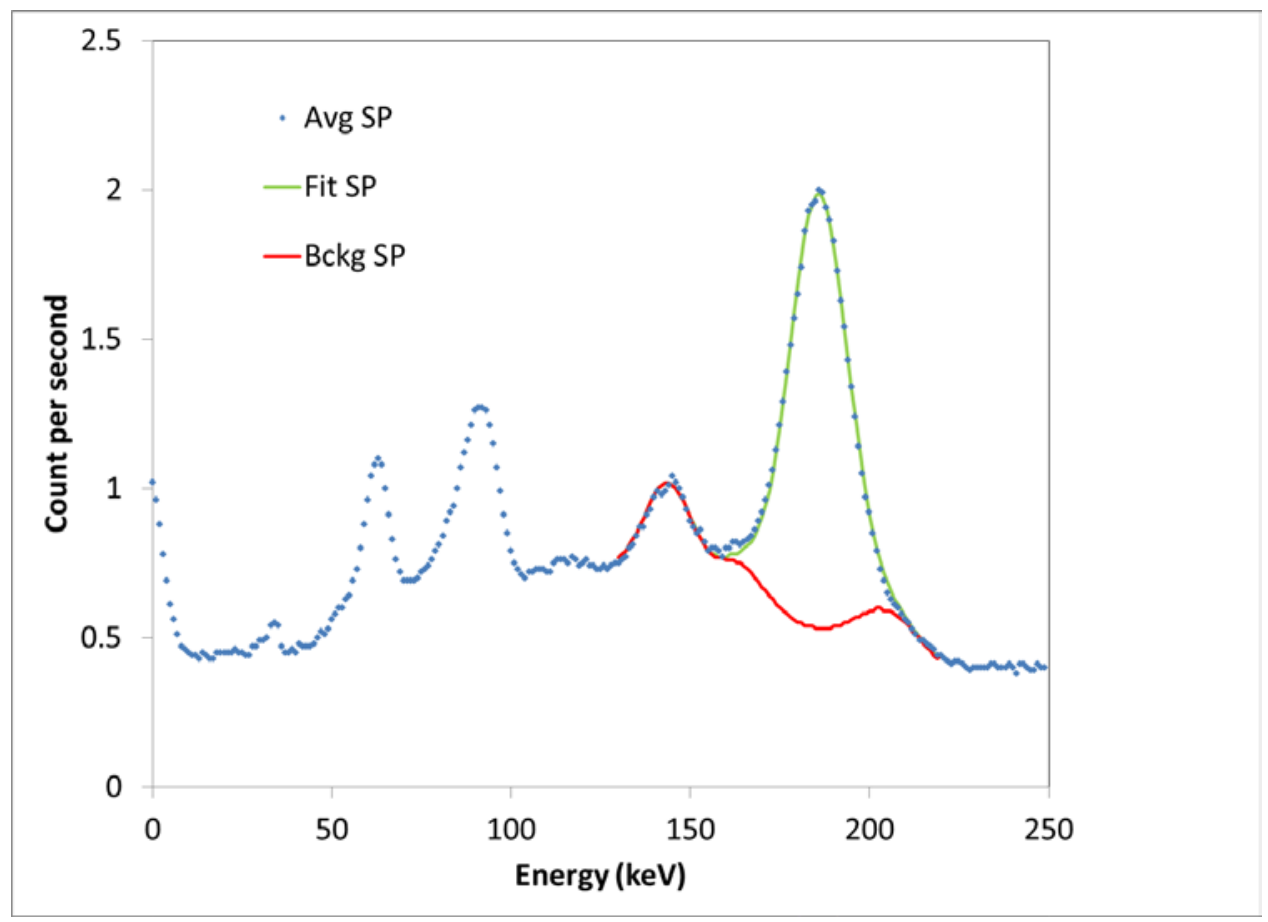

Figure 18. Illustration of Algorithm $05{ }^{235} \mathrm{U}$ spectrum fit process

\subsection{ADAPTIVE BACKGROUND IDENTIFICATION}

If sufficient pressure changes are measured, OLEM can identify the room and deposit backgrounds using an adaptive technique. The OLEM software can calculate two distinct backgrounds: a bin-wise background, and the algorithm-ROI background (Alg01, Alg02, and Alg05 only).

The bin-wise background is generated independently of the algorithm, and it is not used in the calculation of enrichment. Its primary use is a visual confirmation of the data properties and the environment characteristics. It is estimated from a set of measurements at different pressures using the following equation, where $\rho$ is the gas density

$$
S(E)=B(E)+\rho G(E)
$$

A linear regression fit is used for every energy bin to identify the background, $B(E)$, and the gasdependent portion of the spectrum, $\mathrm{G}(\mathrm{E})$. 
The algorithm ROI background is estimated from a linear regression fit as follows

$$
\mathrm{ROI}_{\mathrm{alg}}[\mathrm{S}(\mathrm{E})]=\mathrm{ROI}_{\mathrm{alg}}[\mathrm{B}(\mathrm{E})]+\rho \mathrm{ROI}_{\mathrm{alg}}[\mathrm{G}(\mathrm{E})]
$$

Where $\mathrm{ROI}_{\mathrm{alg}}$ is a function that processes the spectra to generate an ROI "count" and each algorithm uses its own function. Thus, the algorithm background is a single number. The value $\mathrm{ROI}_{\text {alg }}[\mathrm{G}(\mathrm{E})]$ is proportional to the enrichment, $\varepsilon$, through a simple proportionality constant that we call the "enrichment constant," kenrich

$$
\mathrm{ROI}_{\mathrm{alg}}[\mathrm{G}(\mathrm{E})]=\mathrm{k}_{\text {enrich }} \times \varepsilon
$$

To demonstrate the process, we have generated two special cases of data with known backgrounds. The first set of 100-hour worth of data imposes a background with sinusoidal shape; the second imposes a step background. The simulated enrichment was $5 \%$ with the standard 10-hour 10-50 Torr ramp.

Table 2 shows the analysis results with good agreement with data and low $(<1 \%)$ error. It also shows the algorithm backgrounds calculated for each case. As expected Alg01 and Alg02 do a better job than Alg00. Figure 19 and Figure 20 show the analysis results, including the bin-wise background, with a 10-hour background collection time. We conclude that the background identification methodology yields the expected results.

\begin{tabular}{|c|c|c|c|c|c|c|c|}
\hline & \multicolumn{2}{|c|}{$5 \%$ step background } & & & \multicolumn{3}{|c|}{$5 \%$ cosine background } \\
\hline Algorithm & 00 & 01 & 02 & Algorithm & 00 & 01 & 02 \\
\hline Enrichment Avg & 4.98 & 5.00 & 5.00 & Enrichment Avg & 5.00 & 5.00 & 5.01 \\
\hline Enrichment Std. Dev. & $0.65 \%$ & $0.26 \%$ & $0.24 \%$ & Enrichment Std. Dev. & $0.79 \%$ & $0.32 \%$ & $0.22 \%$ \\
\hline Background Avg & 2.33 & 6.23 & 14.61 & Background Avg & 0.89 & 9.68 & 19.38 \\
\hline Background Std. Dev. & $5.50 \%$ & $1.43 \%$ & $0.88 \%$ & Background Std. Dev. & $17.07 \%$ & $1.19 \%$ & $0.65 \%$ \\
\hline
\end{tabular}

Table 2. Analysis results for the special background cases 


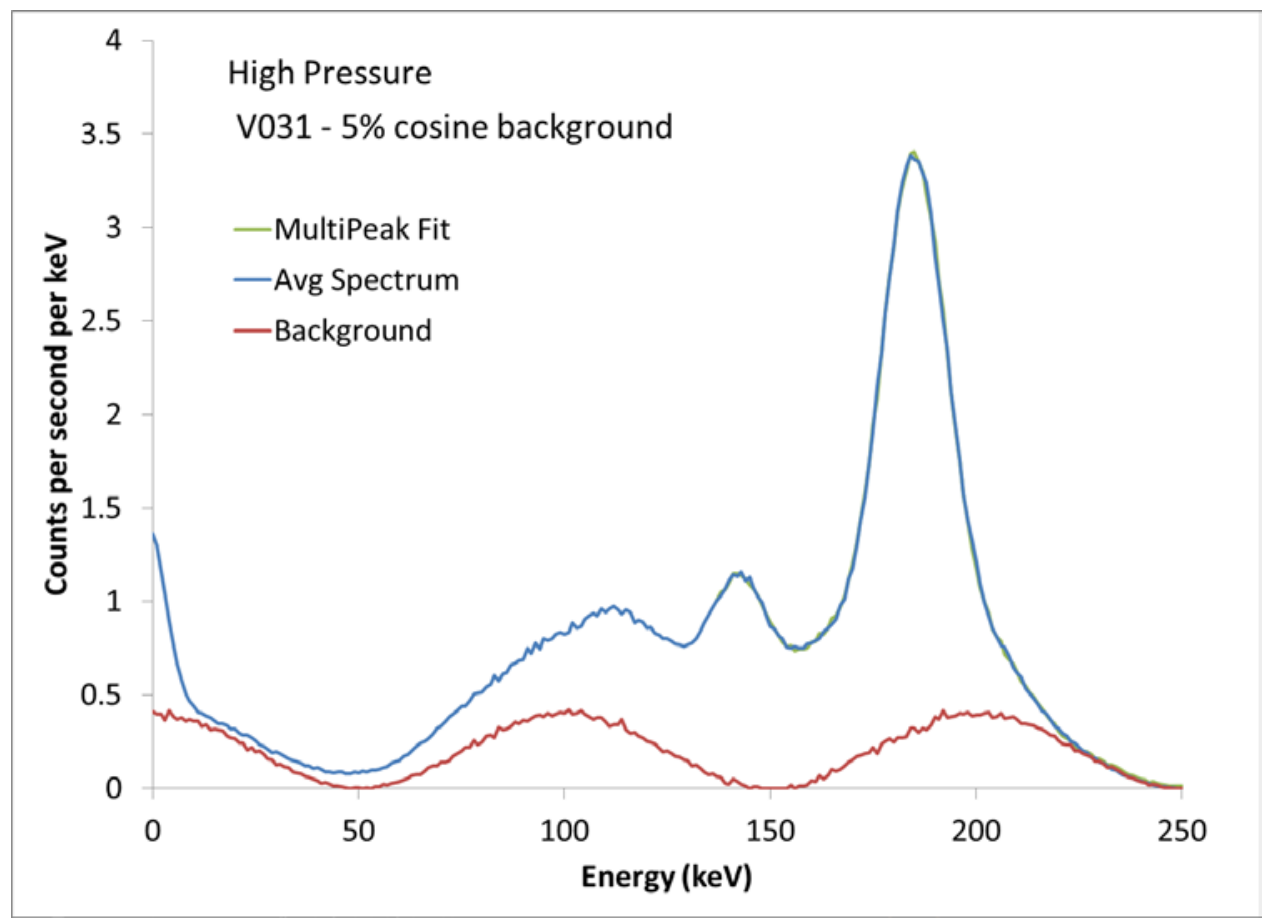

Figure 19. Spectral decomposition for case with sinusoidal background

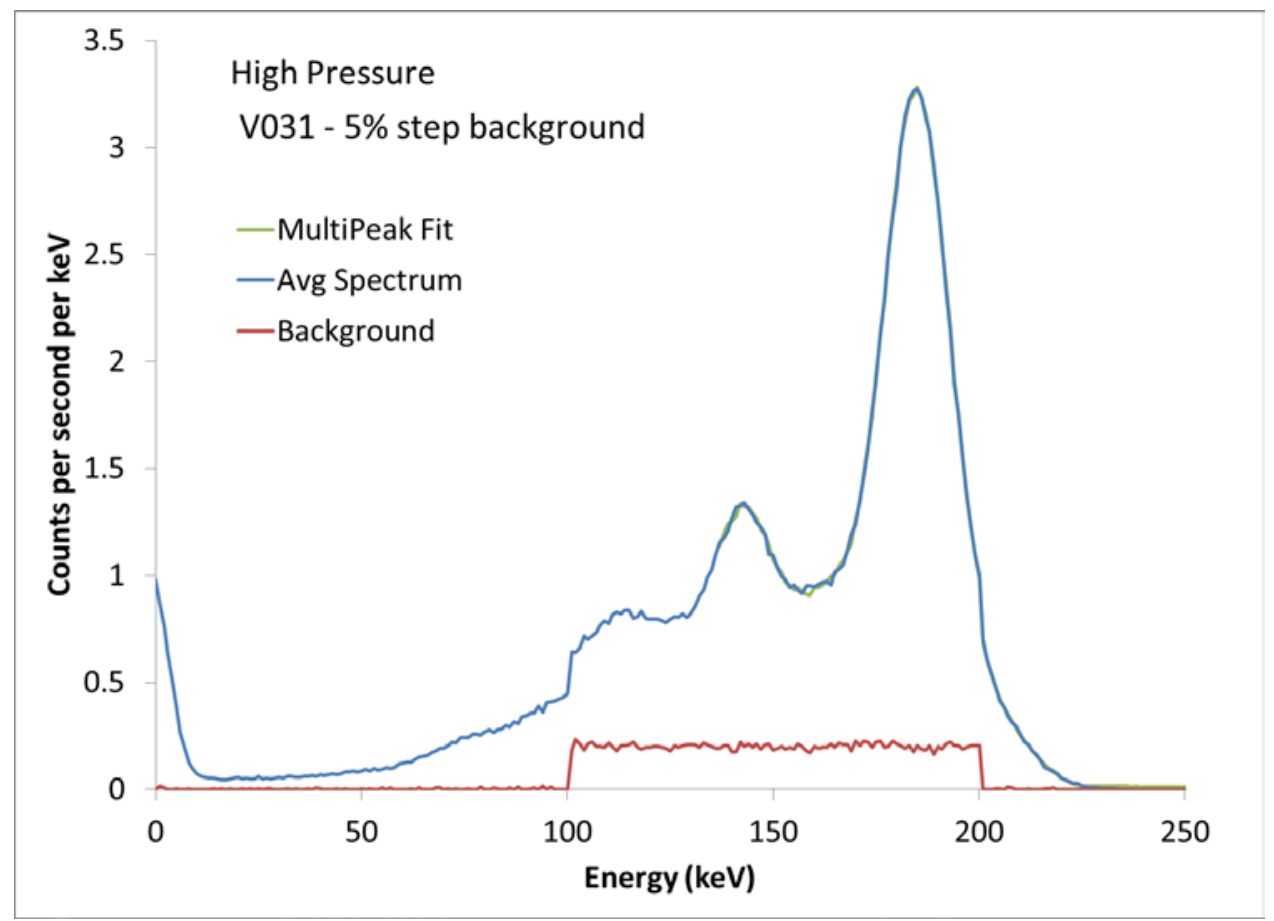

Figure 20. Spectral decomposition for case with step background 


\subsection{RESULTS}

Note: these results (Revision 1 of this document) apply to software Version 866 (2015/11/09 14:57:58)

\subsection{ALGORITHM SETTINGS}

Table 3 through Table 8 document the algorithm setting values used for these analyses. Of special relevance are the ROI limits (chMin and chMax) used. Alg00 and Alg01 use the small $\mathrm{ROI}$, the other algorithms use the large ROI. The enrichment constant is a function of these settings. Note that algorithm 3 was not implemented for this exercise.

Table 3. Algorithm 0 settings

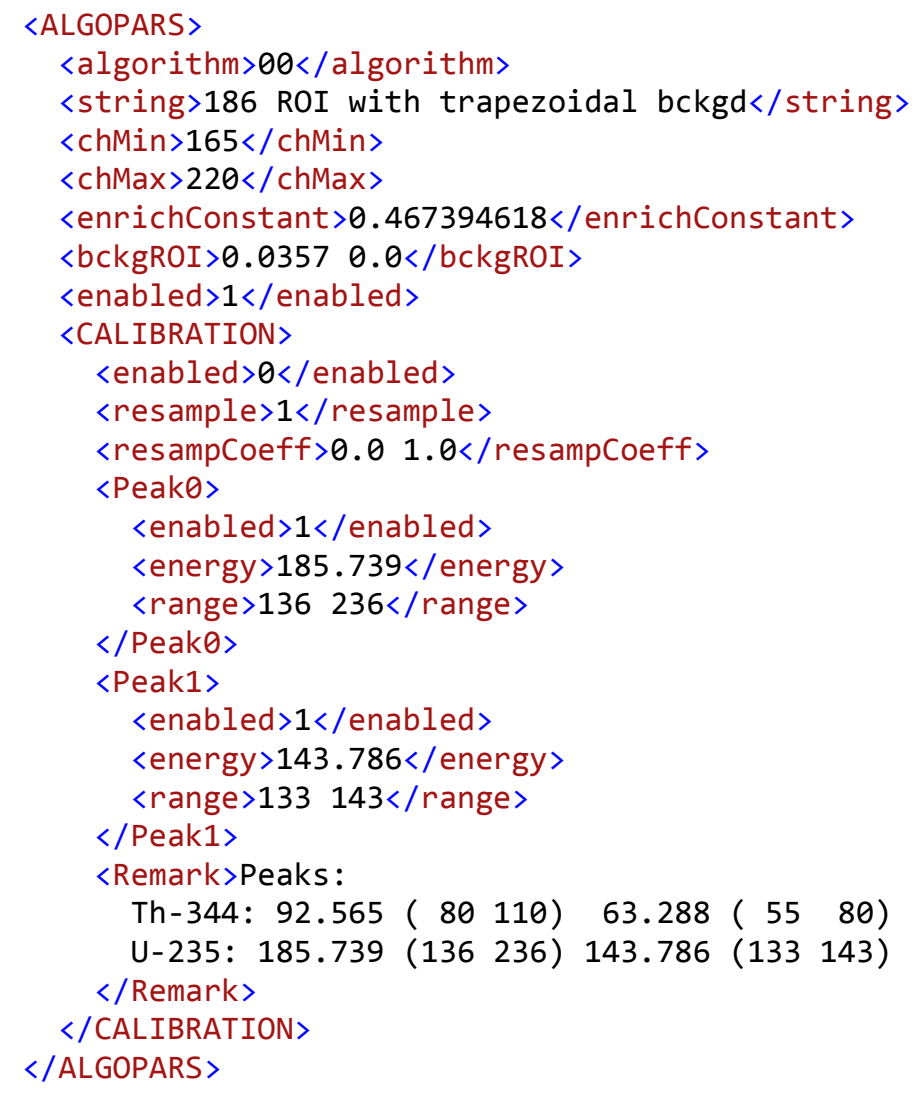


Table 4. Algorithm 1 settings

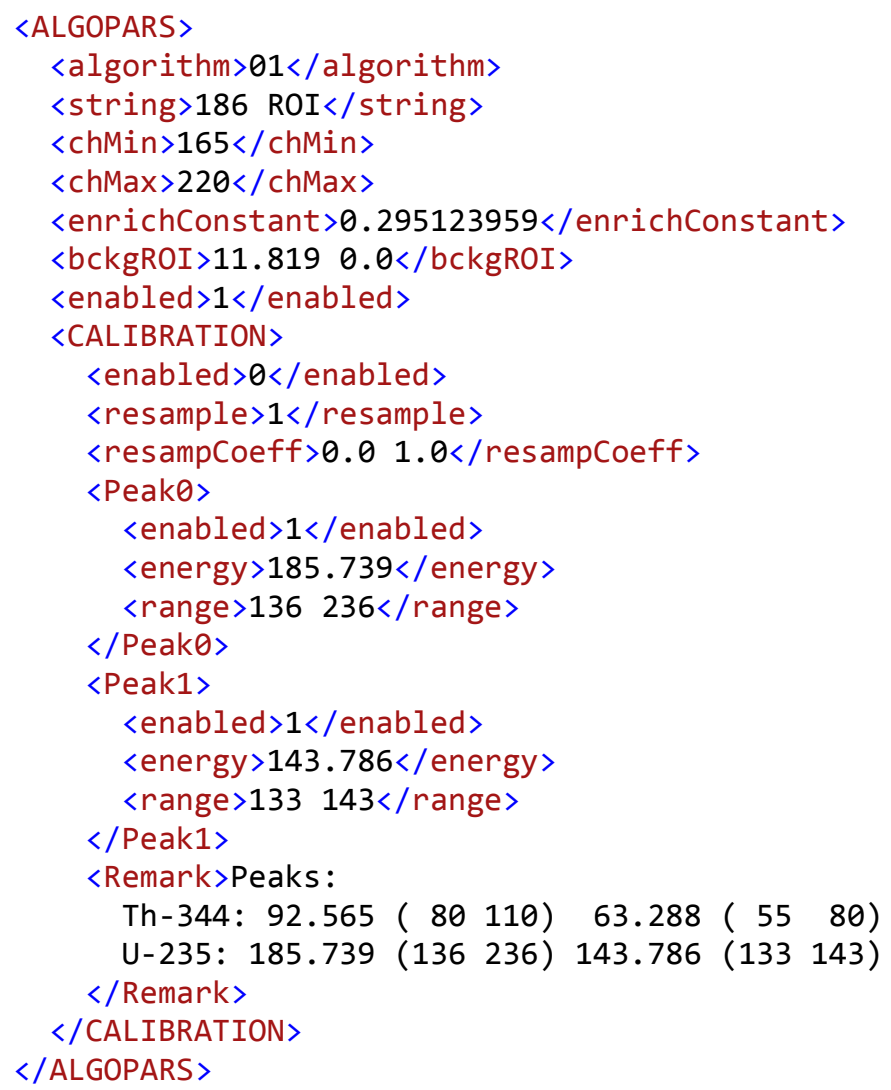


Table 5. Algorithm 2 settings

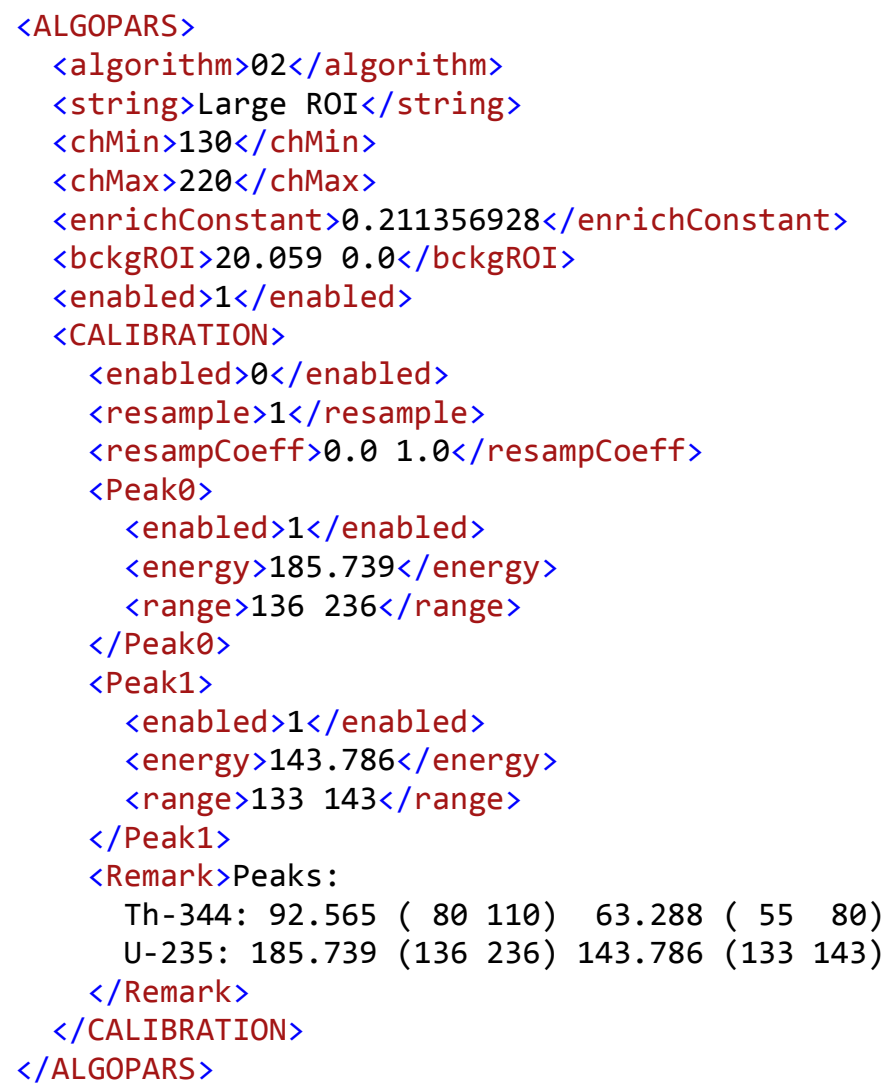

Table 6. Algorithm 3 settings

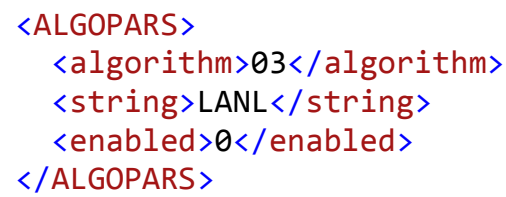


Table 7. Algorithm 4 settings

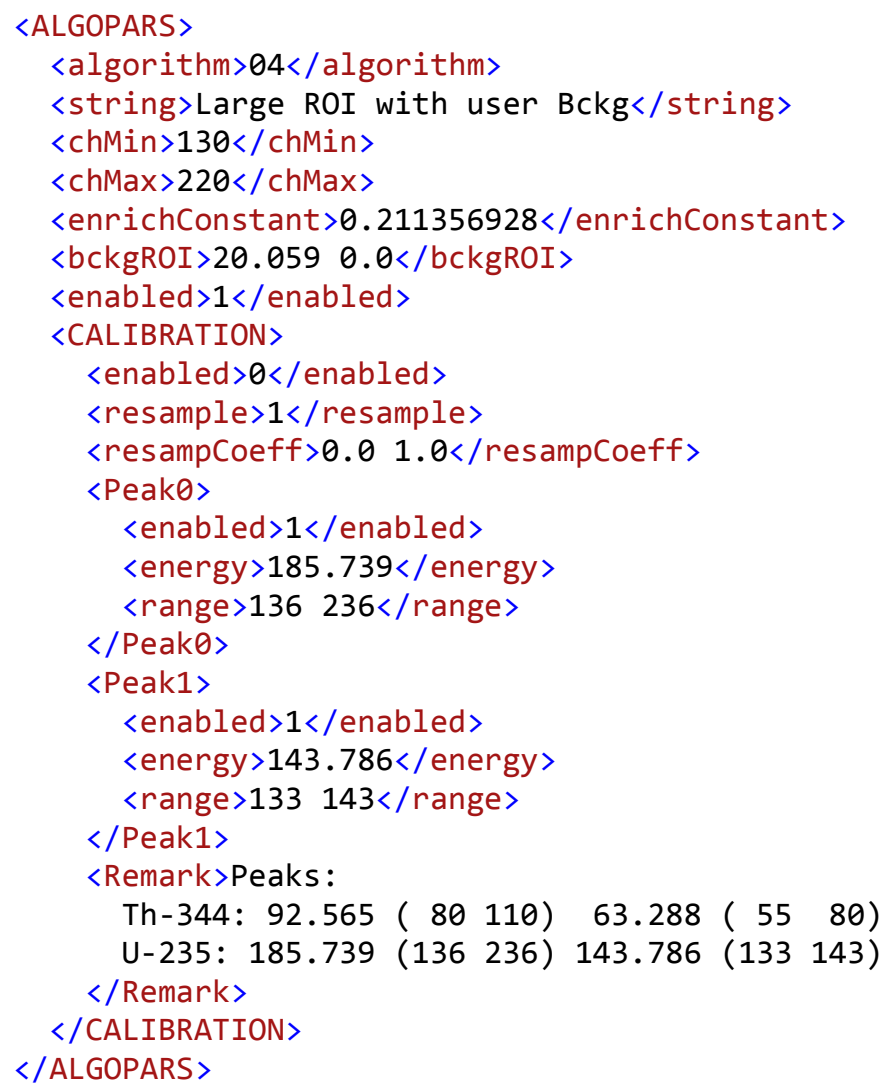


Table 8. Algorithm 5 settings

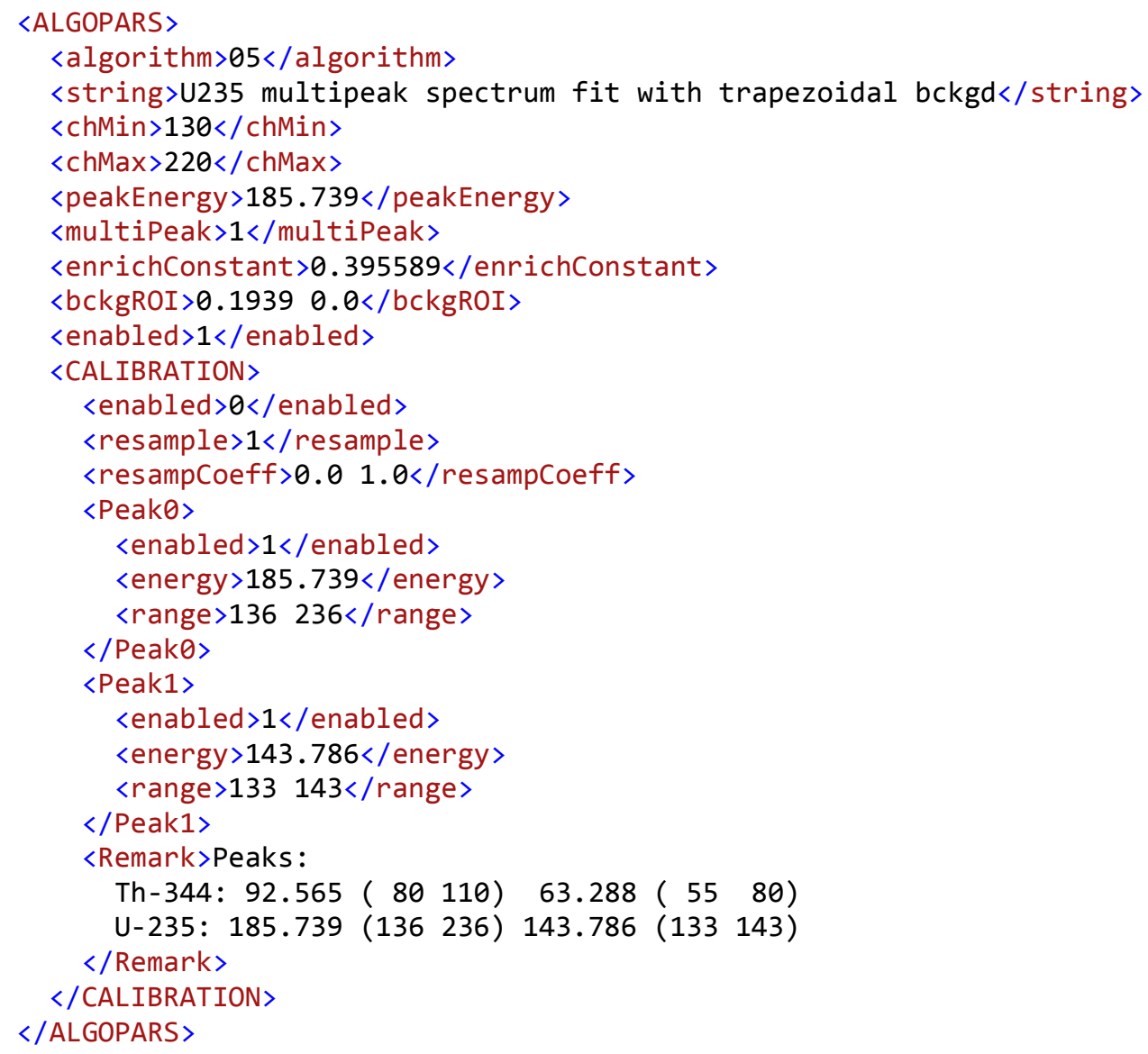

\subsection{MEASURED ENRICHMENT CONSTANT - BEST ESTIMATE USING 4.62\% ENRICHMENT DATA}

The $4.62 \%$ data set is the most consistent measurement and has the most pressure points. For this reason, the $4.62 \%$ set is used to define the enrichment constants, $\mathrm{K}$, that are documented in Table 9. The additional data sets at other enrichments are used to verify the best-estimate values. The units of the enrichment constant are \%enrichment per $\mathrm{cps} /\left(\mathrm{UF} 6 \mathrm{~kg} / \mathrm{m}^{3}\right)$. For a measured count rate of $C$ cps and a UF6 density of $D \mathrm{~kg} / \mathrm{m}^{3}$, the enrichment in percent is calculated as

$$
E(\%)=K * C / D
$$

Table 9. Measured enrichment constants

\begin{tabular}{|r|l|l|l|}
\hline Algo & \multicolumn{3}{|c|}{ Enrichment Constant (\%_enrich) $\mathbf{( U F 6 ~ k g / m 3 ) / c p s ~}$} \\
\hline 0 & $0.467395 \pm$ & 0.006183 & \\
\hline 1 & $0.295124 \pm$ & 0.006440 & \\
\hline 2 & $0.211357 \pm$ & 0.004882 & \\
\hline 4 & $0.211357 \pm$ & 0.004882 & \\
\hline 5 & $0.395589 \pm$ & 0.010391 & \\
\hline
\end{tabular}


Figure 21 through Figure 25 show graphically the calculation of the enrichment constant for the $4.62 \%$ data set and all the active algorithms. As we can see in these figures, the enrichment level scales linearly with density. Table 10 shows the numerical values used for the calculation.

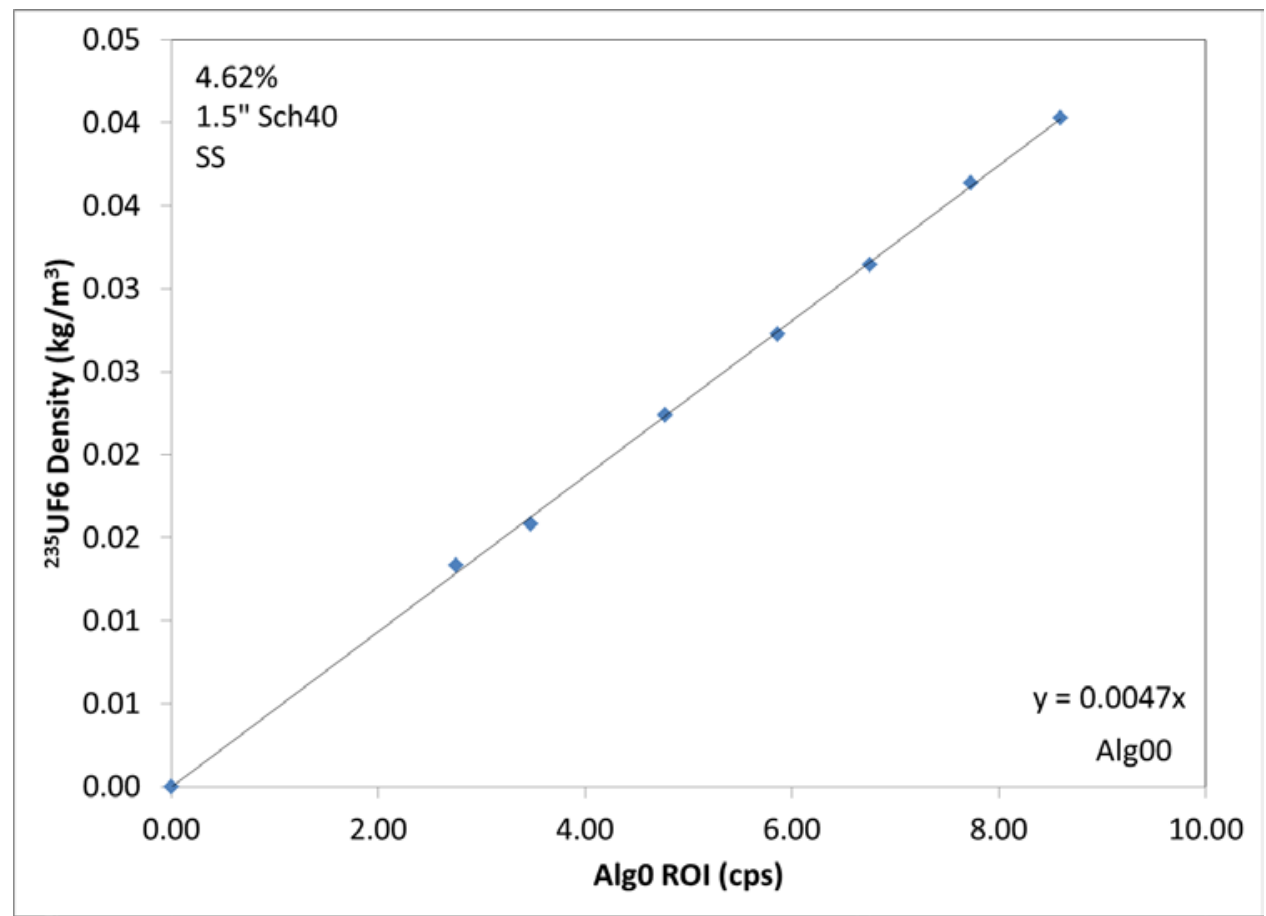

Figure 21. Enrichment constant calculation for Alg00 


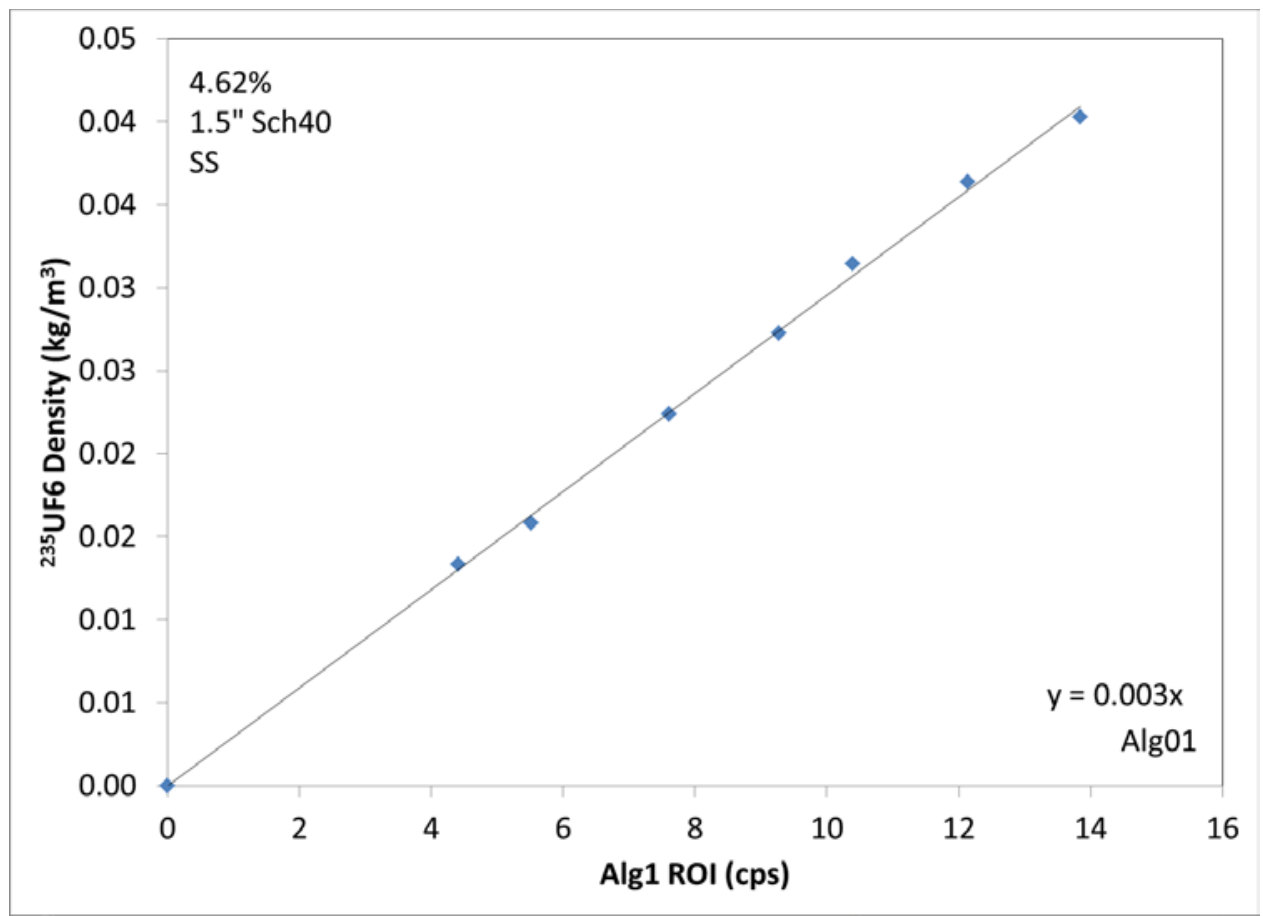

Figure 22. Enrichment constant calculation for Alg01

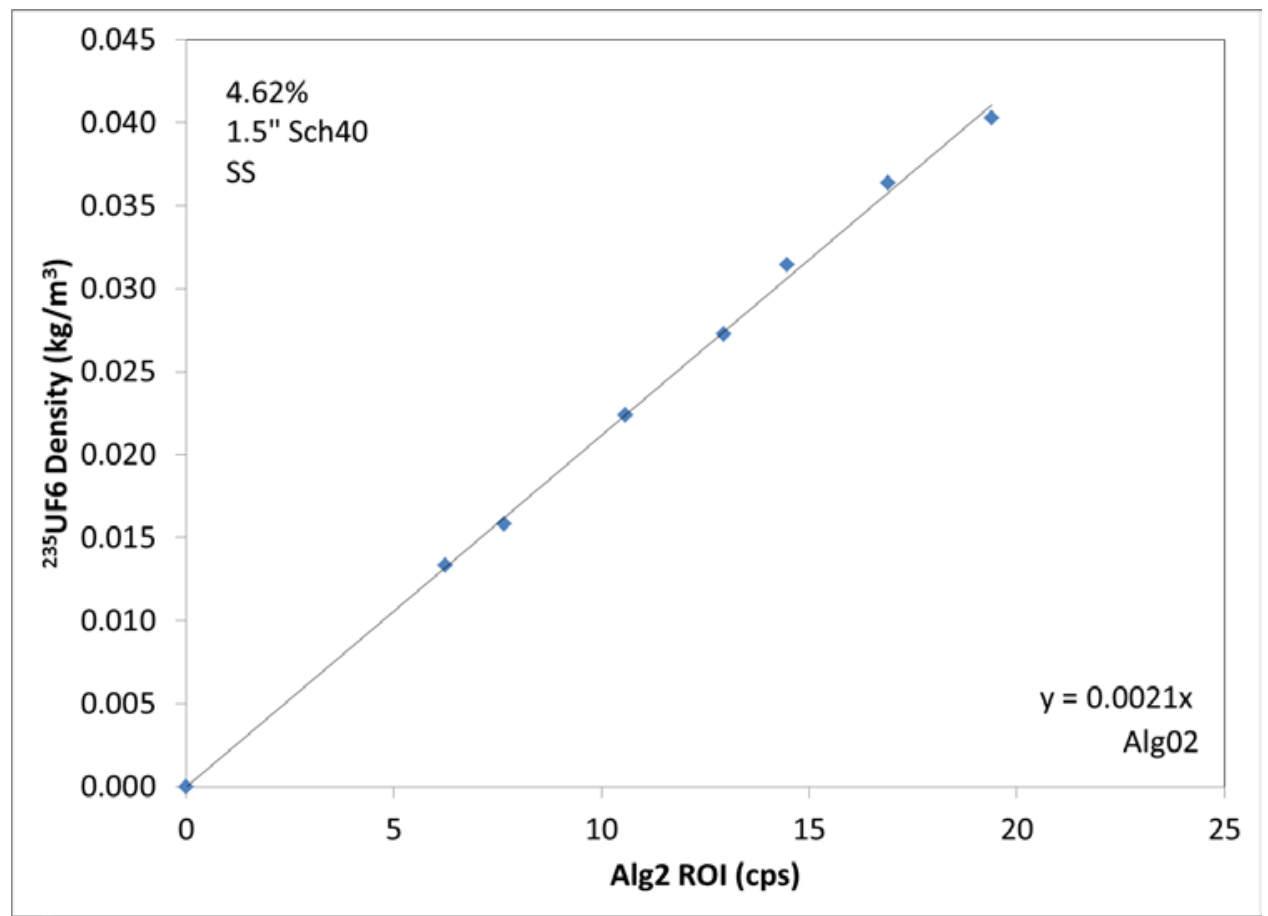

Figure 23. Enrichment constant calculation for Alg02 


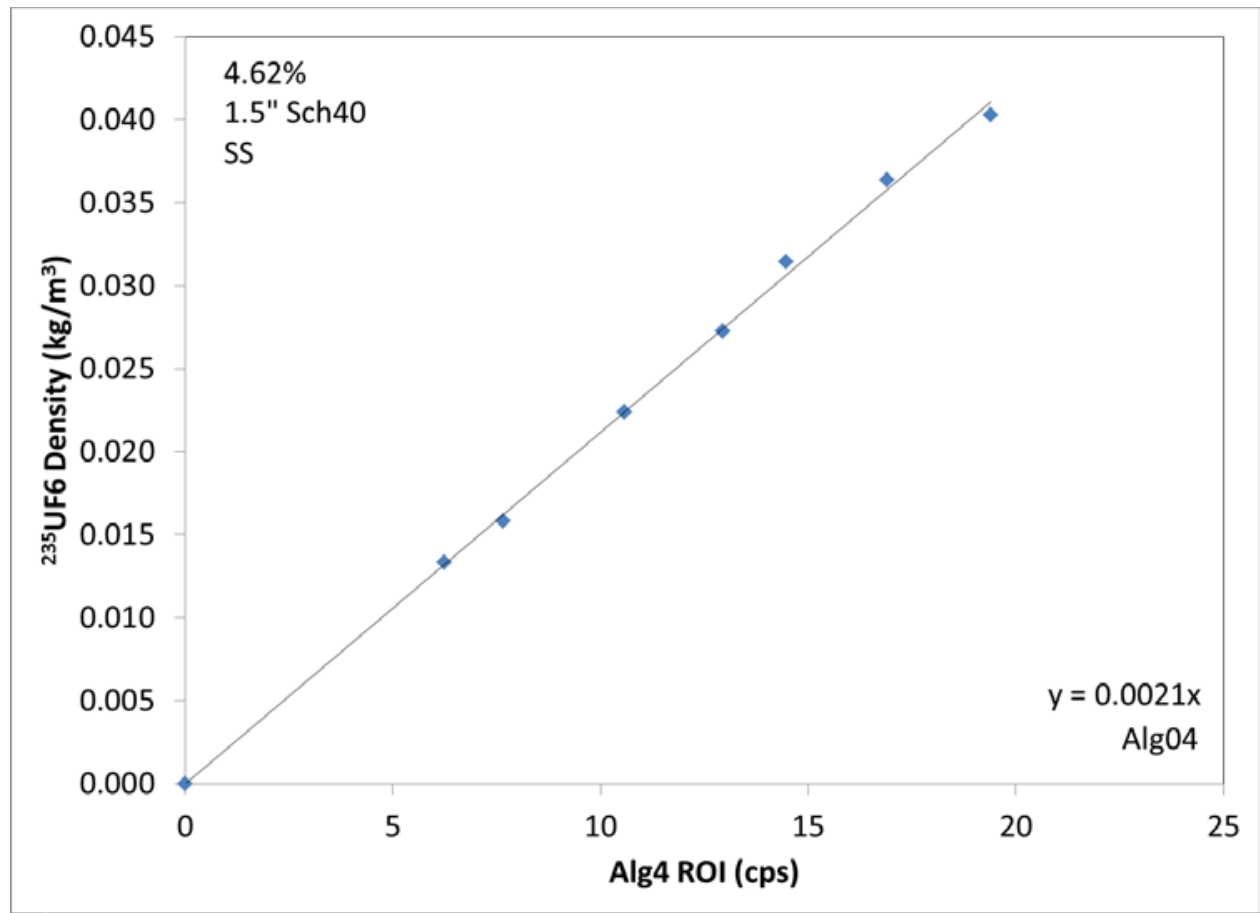

Figure 24. Enrichment constant calculation for Alg04

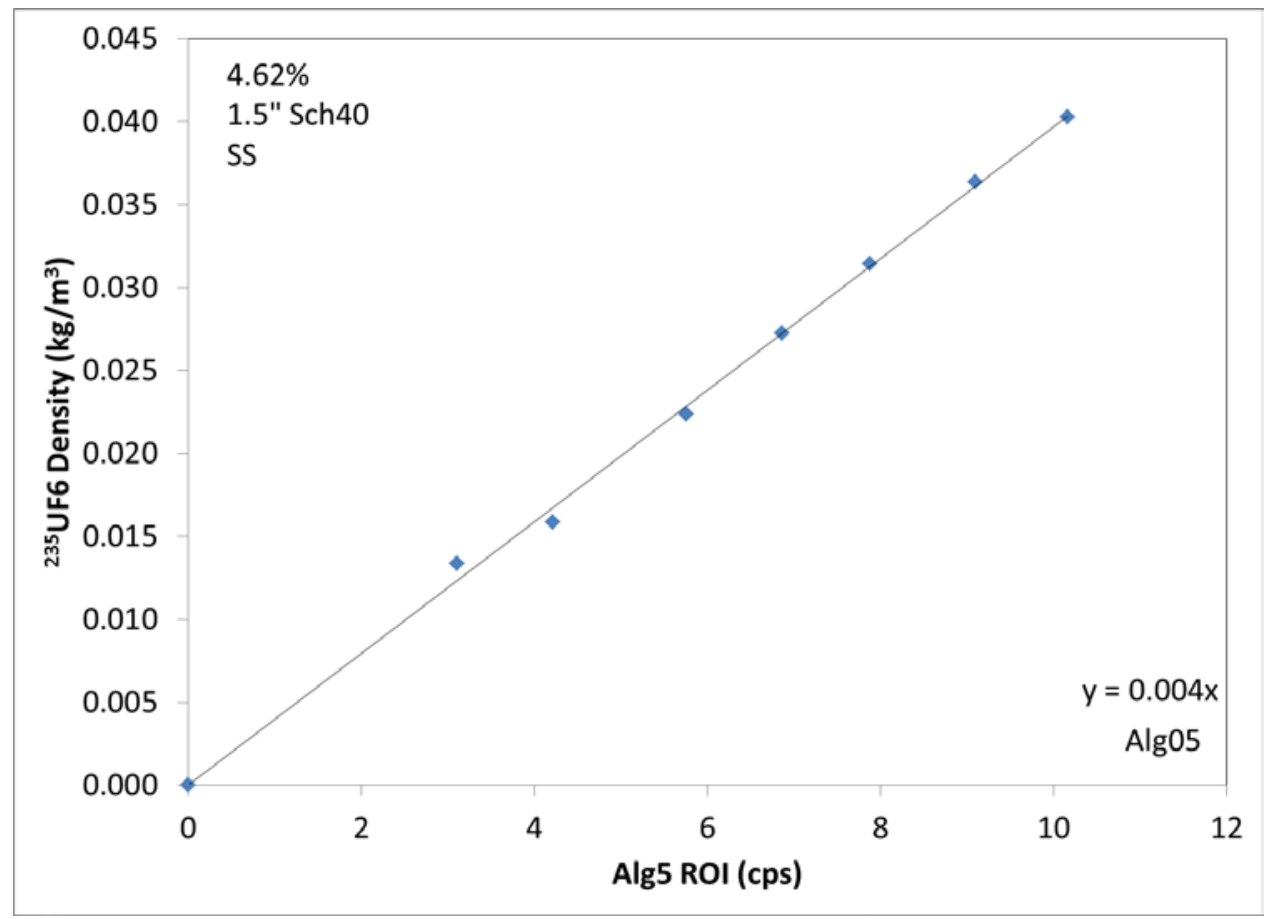

Figure 25. Enrichment constant calculation for Alg05 
Table 10. Measured ROIs for each algorithm, enrichment, and pressure

\begin{tabular}{|c|c|c|c|c|}
\hline \multirow[t]{2}{*}{ iAlgo } & & $\begin{array}{l}\text { 235UF6 } \\
\text { Density } \\
(\mathrm{kg} / \mathrm{m} 3)\end{array}$ & Net ROI & \multirow[t]{2}{*}{ std } \\
\hline & & 0 & 0 & \\
\hline \multirow[t]{20}{*}{0} & \multirow[t]{7}{*}{$4.62 \%$} & 0.013 & 2.749 & 0.050 \\
\hline & & 0.016 & 3.478 & 0.046 \\
\hline & & 0.022 & 4.776 & 0.045 \\
\hline & & 0.027 & 5.863 & 0.020 \\
\hline & & 0.031 & 6.757 & 0.053 \\
\hline & & 0.036 & 7.727 & 0.039 \\
\hline & & 0.040 & 8.596 & 0.021 \\
\hline & \multirow[t]{4}{*}{$0.71 \%$} & 0.003 & 0.647 & 0.033 \\
\hline & & 0.005 & 1.140 & 0.033 \\
\hline & & 0.007 & 1.414 & 0.017 \\
\hline & & 0.001 & 0.257 & 0.015 \\
\hline & \multirow[t]{3}{*}{$2.97 \%$} & 0.014 & 3.101 & 0.026 \\
\hline & & 0.024 & 5.407 & 0.039 \\
\hline & & 0.004 & 0.915 & 0.016 \\
\hline & \multirow[t]{3}{*}{$93.70 \%$} & 0.200 & 45.771 & 0.104 \\
\hline & & 0.266 & 60.876 & 0.109 \\
\hline & & 0.112 & 25.707 & 0.104 \\
\hline & \multirow[t]{4}{*}{$6.00 \%$} & 0.033 & 7.374 & 0.062 \\
\hline & & 0.066 & 14.097 & 0.050 \\
\hline & & 0.011 & 2.203 & 0.095 \\
\hline & & 0.000 & 0.000 & \\
\hline \multirow[t]{12}{*}{1} & \multirow[t]{7}{*}{$4.62 \%$} & 0.013 & 4.407 & 0.050 \\
\hline & & 0.016 & 5.515 & 0.046 \\
\hline & & 0.022 & 7.610 & 0.045 \\
\hline & & 0.027 & 9.270 & 0.020 \\
\hline & & 0.031 & 10.395 & 0.053 \\
\hline & & 0.036 & 12.135 & 0.039 \\
\hline & & 0.040 & 13.843 & 0.021 \\
\hline & \multirow[t]{4}{*}{$0.71 \%$} & 0.003 & 0.909 & 0.033 \\
\hline & & 0.005 & 1.545 & 0.033 \\
\hline & & 0.007 & 2.150 & 0.017 \\
\hline & & 0.001 & 0.438 & 0.015 \\
\hline & $2.97 \%$ & 0.014 & 4.559 & 0.026 \\
\hline
\end{tabular}




\begin{tabular}{|c|c|c|c|c|}
\hline & & 0.024 & 8.026 & 0.039 \\
\hline & & 0.004 & 1.372 & 0.016 \\
\hline & $93.70 \%$ & 0.200 & 68.605 & 0.104 \\
\hline & & 0.266 & 90.575 & 0.109 \\
\hline & & 0.112 & 38.180 & 0.104 \\
\hline & $6.00 \%$ & 0.033 & 10.672 & 0.062 \\
\hline & & 0.066 & 20.698 & 0.050 \\
\hline & & 0.011 & 3.301 & 0.095 \\
\hline & & 0.000 & 0.000 & \\
\hline 2 & $4.62 \%$ & 0.013 & 6.238 & 0.064 \\
\hline & & 0.016 & 7.654 & 0.058 \\
\hline & & 0.022 & 10.573 & 0.056 \\
\hline & & 0.027 & 12.940 & 0.025 \\
\hline & & 0.031 & 14.479 & 0.066 \\
\hline & & 0.036 & 16.910 & 0.048 \\
\hline & & 0.040 & 19.389 & 0.027 \\
\hline & $0.71 \%$ & 0.003 & 1.238 & 0.043 \\
\hline & & 0.005 & 2.158 & 0.044 \\
\hline & & 0.007 & 2.975 & 0.022 \\
\hline & & 0.001 & 0.616 & 0.020 \\
\hline & $2.97 \%$ & 0.014 & 6.329 & 0.033 \\
\hline & & 0.024 & 11.129 & 0.049 \\
\hline & & 0.004 & 1.900 & 0.020 \\
\hline & $93.70 \%$ & 0.200 & 100.879 & 0.124 \\
\hline & & 0.266 & 131.348 & 0.130 \\
\hline & & 0.112 & 58.718 & 0.126 \\
\hline & $6.00 \%$ & 0.033 & 14.670 & 0.078 \\
\hline & & 0.066 & 28.543 & 0.061 \\
\hline & & 0.011 & 4.572 & 0.121 \\
\hline & & 0.000 & 0.000 & \\
\hline 3 & $4.62 \%$ & 0.013 & 0.000 & 0.000 \\
\hline & & 0.016 & 0.000 & 0.000 \\
\hline & & 0.022 & 0.000 & 0.000 \\
\hline & & 0.027 & 0.000 & 0.000 \\
\hline & & 0.031 & 0.000 & 0.000 \\
\hline & & 0.036 & 0.000 & 0.000 \\
\hline & & 0.040 & 0.000 & 0.000 \\
\hline & $0.71 \%$ & 0.000 & 0.000 & 0.000 \\
\hline & & 0.001 & 0.000 & 0.000 \\
\hline
\end{tabular}




\begin{tabular}{|c|c|c|c|c|}
\hline & & 0.003 & 0.000 & 0.000 \\
\hline & & 0.005 & 0.000 & 0.000 \\
\hline & $2.97 \%$ & 0.004 & 0.000 & 0.000 \\
\hline & & 0.014 & 0.000 & 0.000 \\
\hline & & 0.024 & 0.000 & 0.000 \\
\hline & $93.70 \%$ & 0.112 & 0.000 & 0.000 \\
\hline & & 0.200 & 0.000 & 0.000 \\
\hline & & 0.266 & 0.000 & 0.000 \\
\hline & $6.00 \%$ & 0.011 & 0.000 & 0.000 \\
\hline & & 0.033 & 0.000 & 0.000 \\
\hline & & 0.066 & 0.000 & 0.000 \\
\hline & & 0.000 & 0.000 & \\
\hline 4 & $4.62 \%$ & 0.013 & 6.238 & 0.064 \\
\hline & & 0.016 & 7.654 & 0.058 \\
\hline & & 0.022 & 10.573 & 0.056 \\
\hline & & 0.027 & 12.940 & 0.025 \\
\hline & & 0.031 & 14.479 & 0.066 \\
\hline & & 0.036 & 16.910 & 0.048 \\
\hline & & 0.040 & 19.389 & 0.027 \\
\hline & $0.71 \%$ & 0.003 & 1.238 & 0.043 \\
\hline & & 0.005 & 2.158 & 0.044 \\
\hline & & 0.007 & 2.975 & 0.022 \\
\hline & & 0.001 & 0.616 & 0.020 \\
\hline & $2.97 \%$ & 0.014 & 6.329 & 0.033 \\
\hline & & 0.024 & 11.129 & 0.049 \\
\hline & & 0.004 & 1.900 & 0.020 \\
\hline & $93.70 \%$ & 0.200 & 95.089 & 0.124 \\
\hline & & 0.266 & 125.558 & 0.130 \\
\hline & & 0.112 & 52.928 & 0.126 \\
\hline & $6.00 \%$ & 0.033 & 14.670 & 0.078 \\
\hline & & 0.066 & 28.543 & 0.061 \\
\hline & & 0.011 & 4.572 & 0.121 \\
\hline & & 0.000 & 0.000 & \\
\hline 5 & $4.62 \%$ & 0.013 & 3.081 & 0.093 \\
\hline & & 0.016 & 4.093 & 0.109 \\
\hline & & 0.022 & 5.563 & 0.106 \\
\hline & & 0.027 & 6.785 & 0.053 \\
\hline & & 0.031 & 7.737 & 0.119 \\
\hline & & 0.036 & 8.945 & 0.069 \\
\hline
\end{tabular}




$\begin{array}{rrrr} & 0.040 & 9.915 & 0.060 \\ & 0.003 & 0.658 & 0.066 \\ & 0.005 & 1.279 & 0.068 \\ & 0.007 & 1.603 & 0.049 \\ & 0.001 & 0.334 & 0.061 \\ & 0.014 & 3.545 & 0.061 \\ 93.70 \% & 0.024 & 6.221 & 0.069 \\ & 0.004 & 1.060 & 0.053 \\ & 0.200 & 53.786 & 0.290 \\ 6.00 \% & 0.266 & 71.146 & 0.385 \\ & 0.112 & 30.004 & 0.166 \\ & 0.033 & 8.412 & 0.118 \\ & 0.066 & 16.424 & 0.109 \\ & 0.011 & 2.644 & 0.144\end{array}$

\subsection{VERIFICATION OF RESULTS USING ALL DATA SETS}

Section 4.0 documents the measured spectra for all the enrichment levels in all the data sets. These data sets have been plotted against the best-estimate enrichment constants identified using the $4.62 \%$ data set. Figure 26 through Figure 30 shows a comparison of the enrichment constant derived from the $4.62 \%$ data set against the measurements at other enrichments. Note that the $93.7 \%$ data is an outlier in this fit because it produces so many counts; therefore, it weights too much in the fit process. In addition, the $93.7 \%$ data set was measured at the low end of pressure ranges, so the pressure estimation (which has about \pm 1 Torr uncertainty) has a larger relative error. For this reason, the plots in Figure 26 through Figure 30 do not show the $93.7 \%$ data. The comparison against entire data set, including $93.7 \%$, is shown in Figure 31 through Figure 35. 


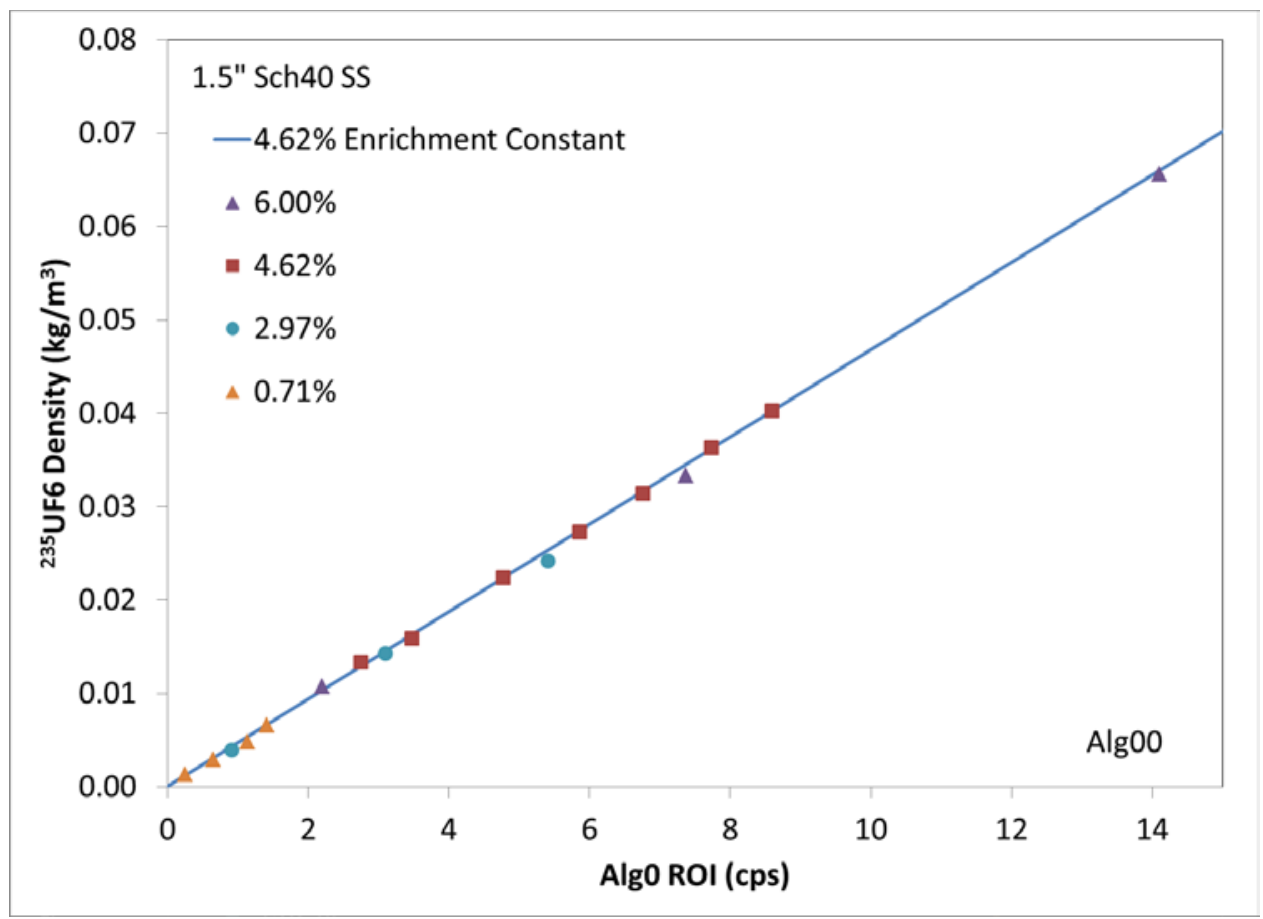

Figure 26. Verification of enrichment constant calculation for Alg00

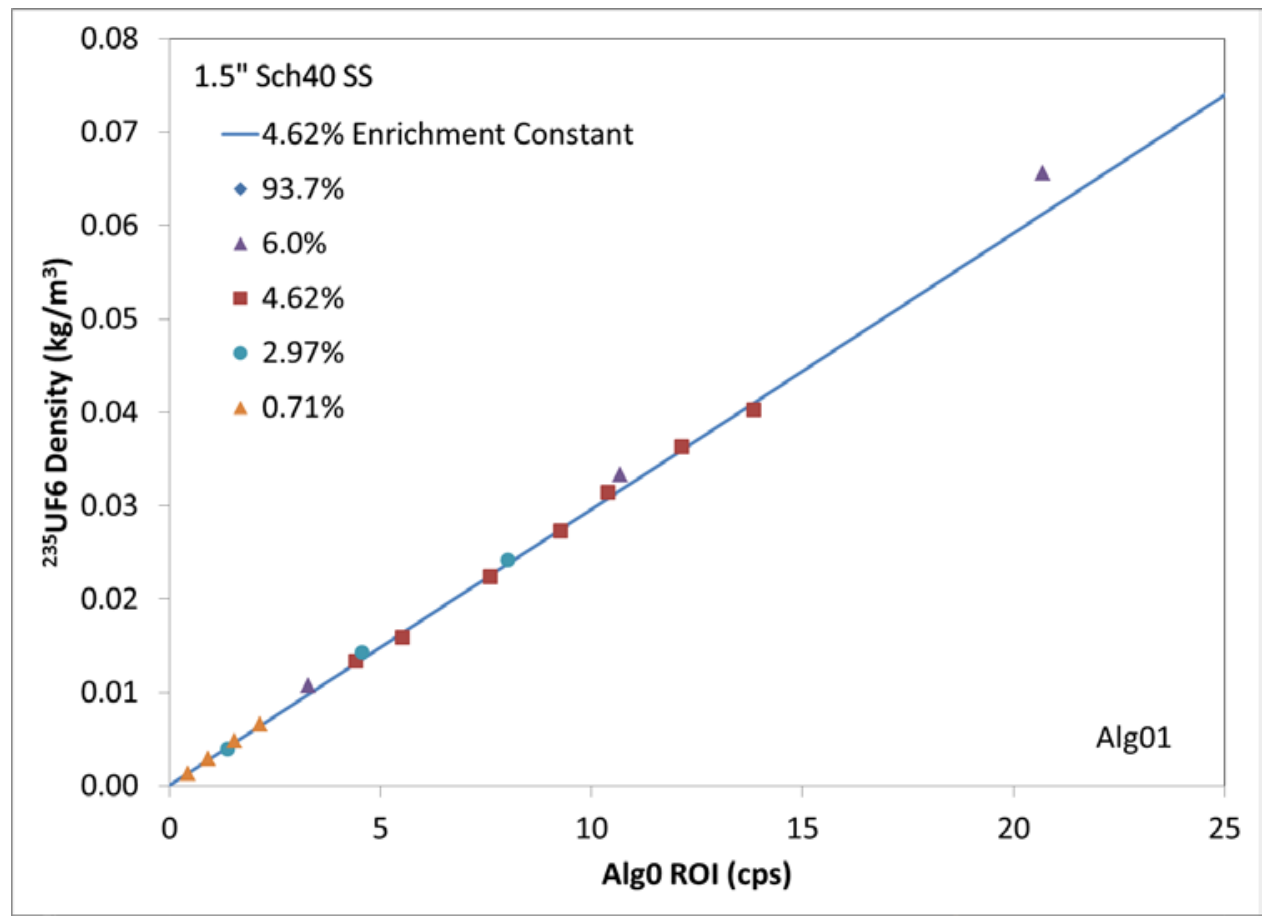

Figure 27. Verification of enrichment constant calculation for Alg01 


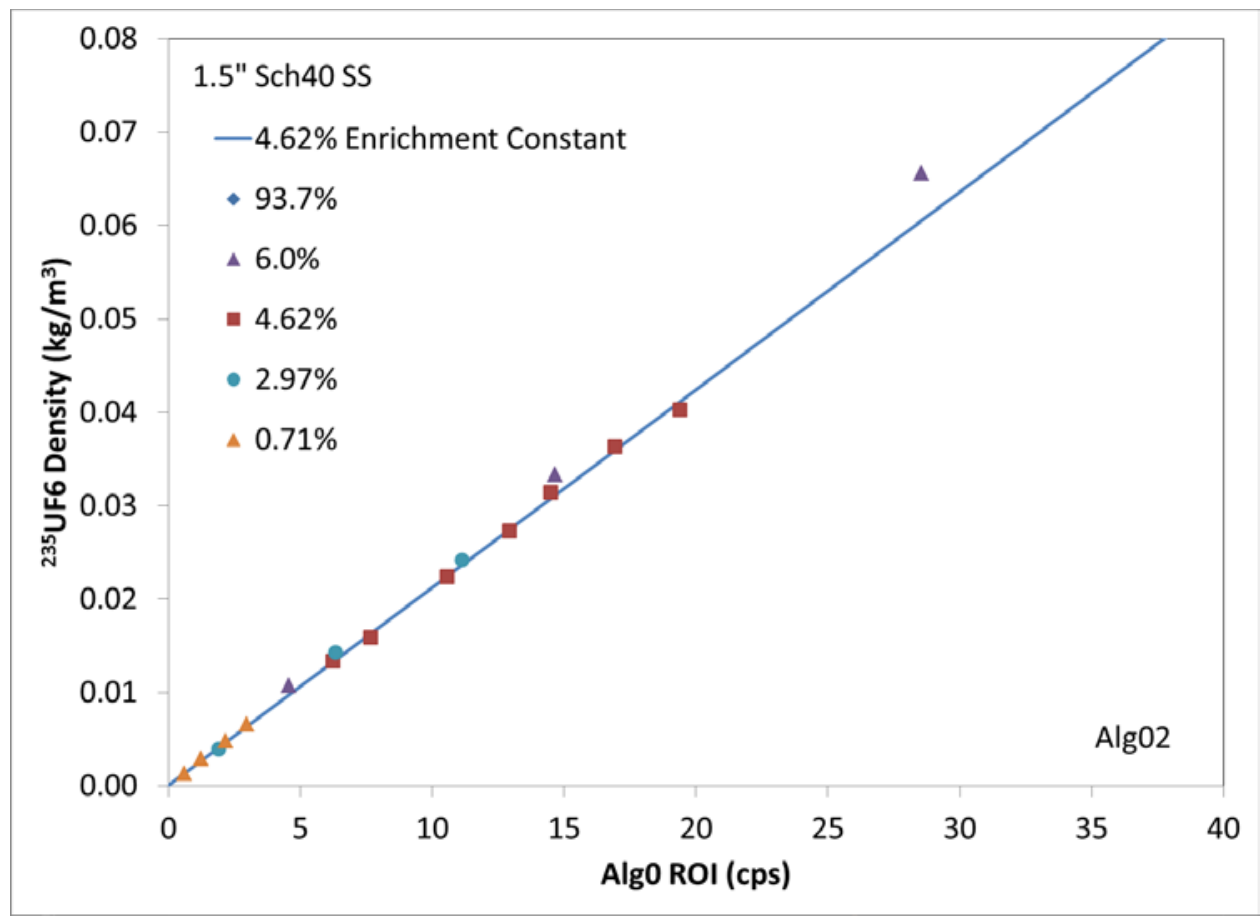

Figure 28. Verification of enrichment constant calculation for Alg02

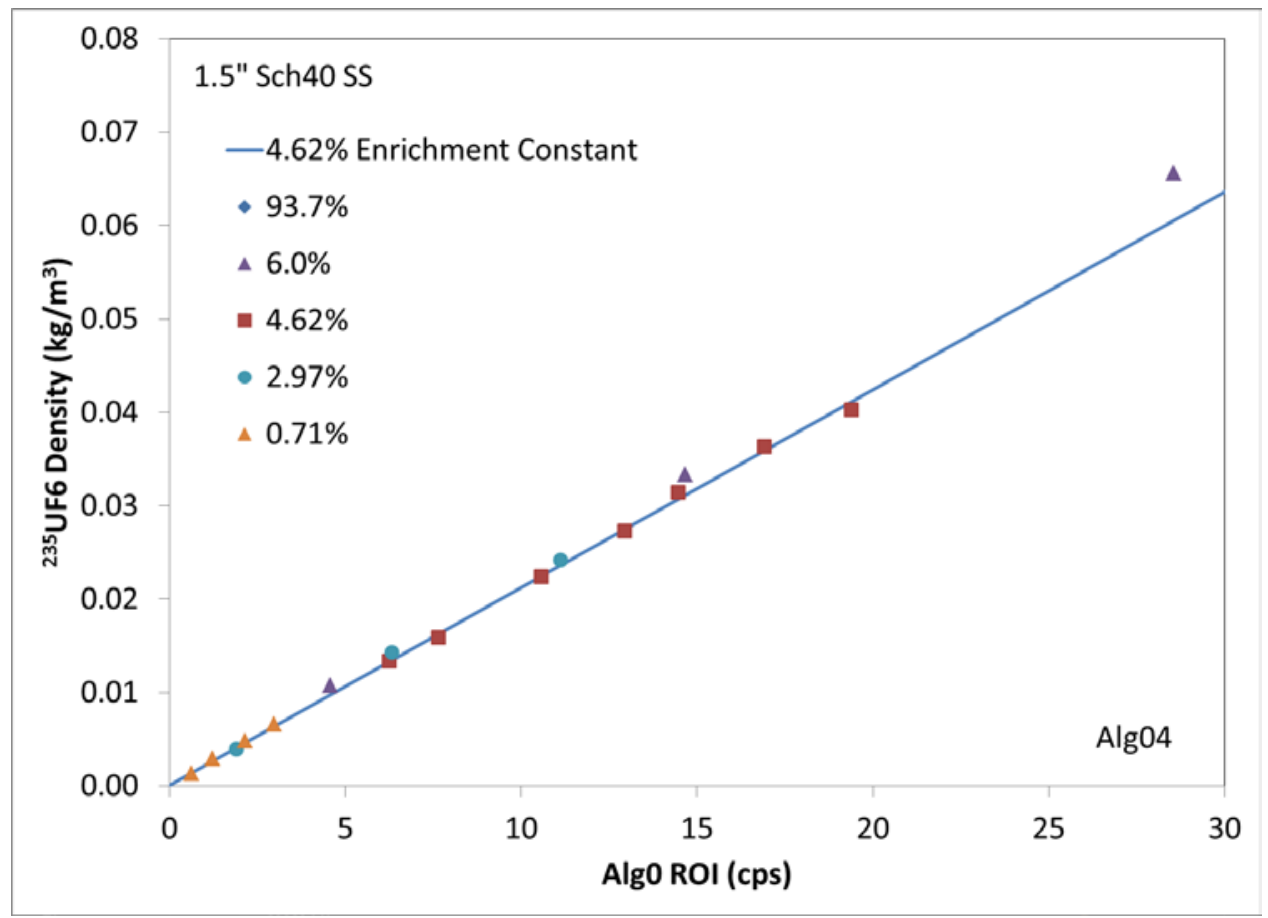

Figure 29. Verification of enrichment constant calculation for Alg04 


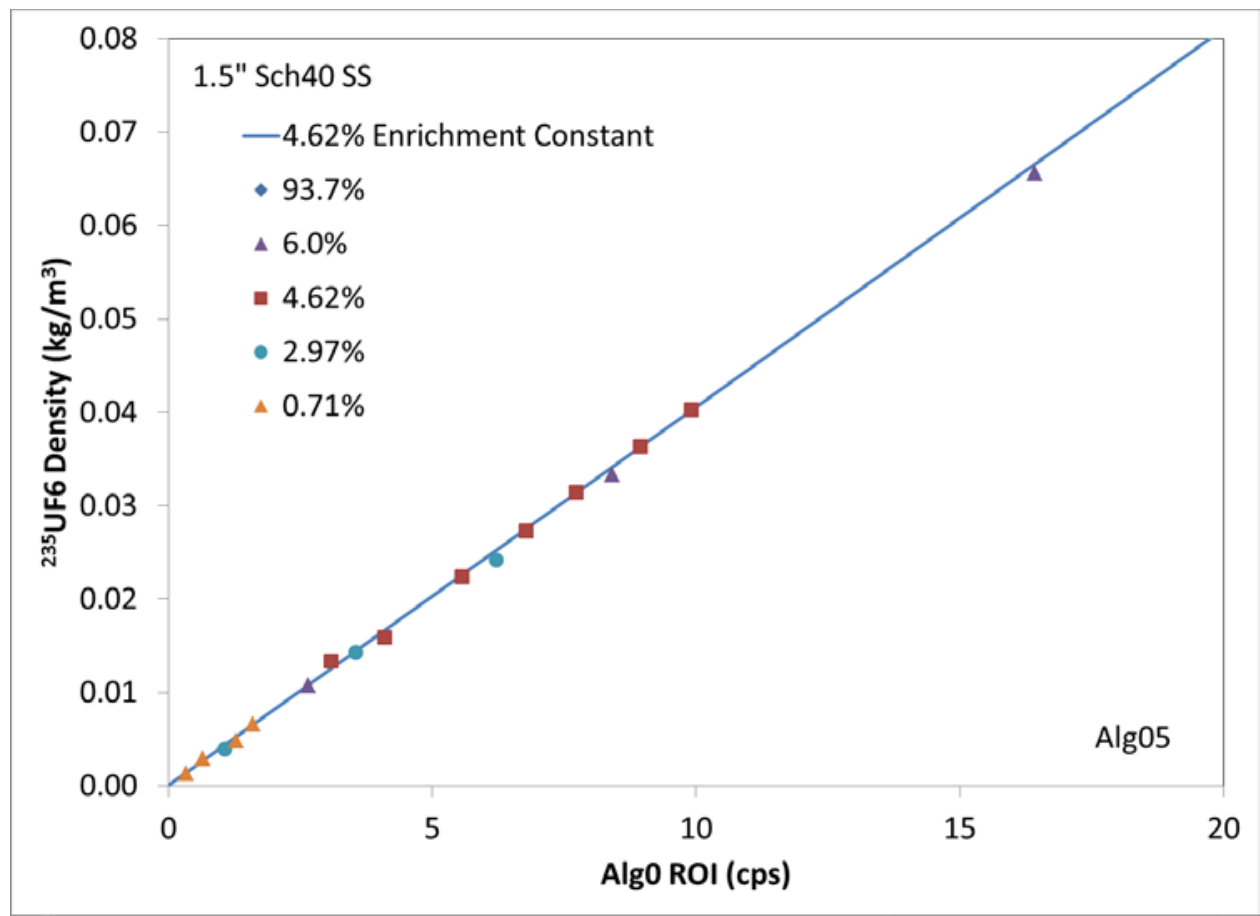

Figure 30. Verification of enrichment constant calculation for Alg05

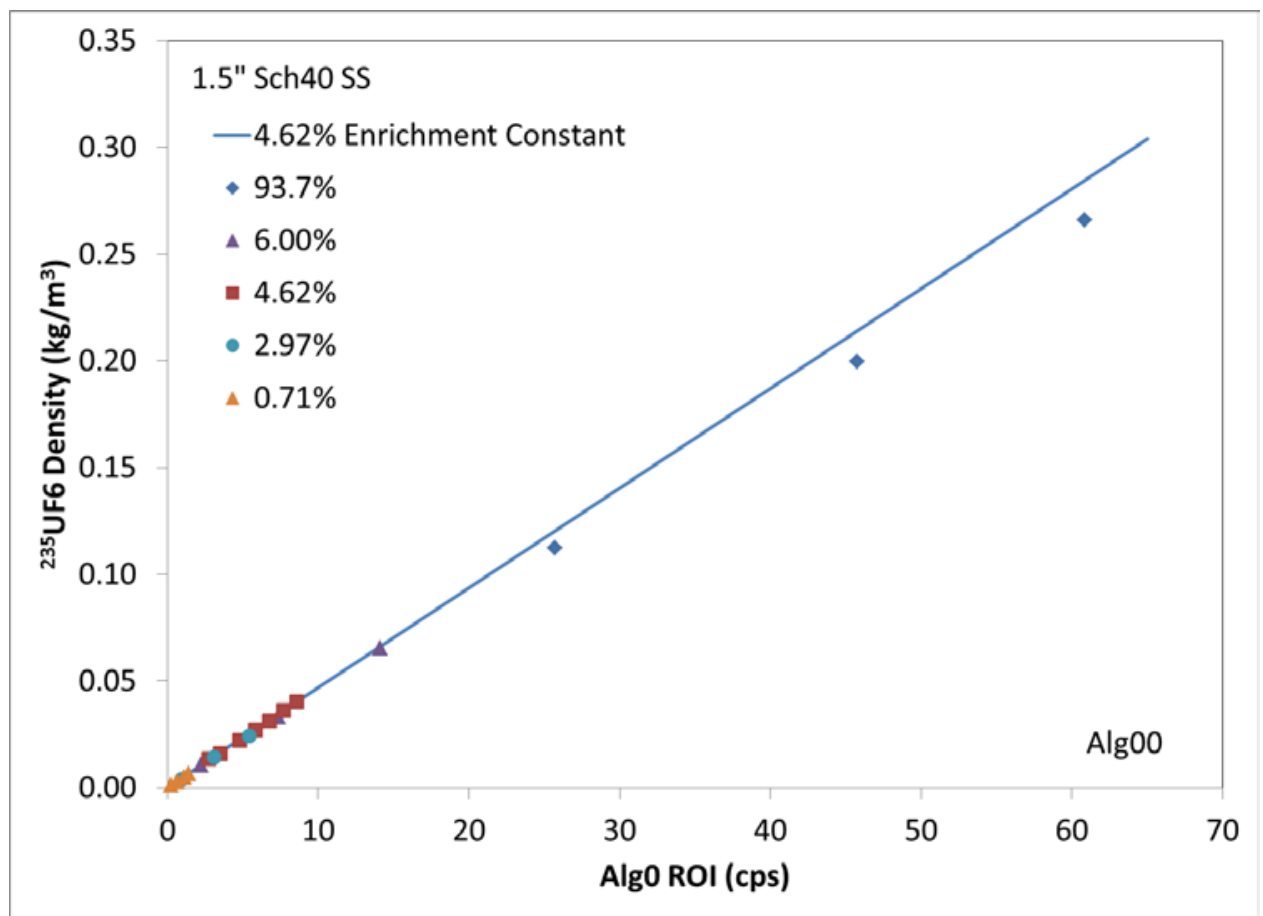

Figure 31. Verification of enrichment constant calculation for Alg00, including the $93.7 \%$ data set 


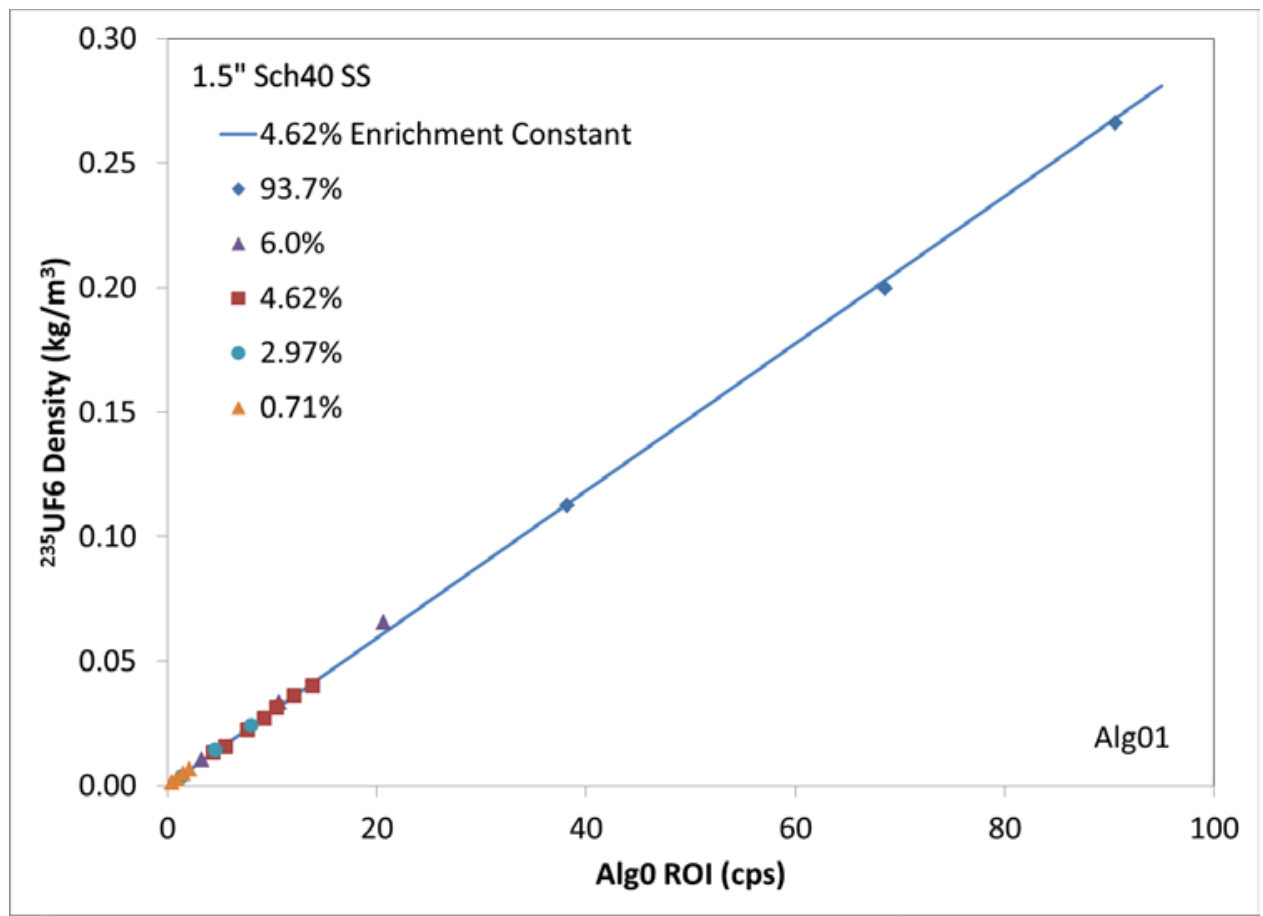

Figure 32. Verification of enrichment constant calculation for Alg01, including the 93.7\% data set

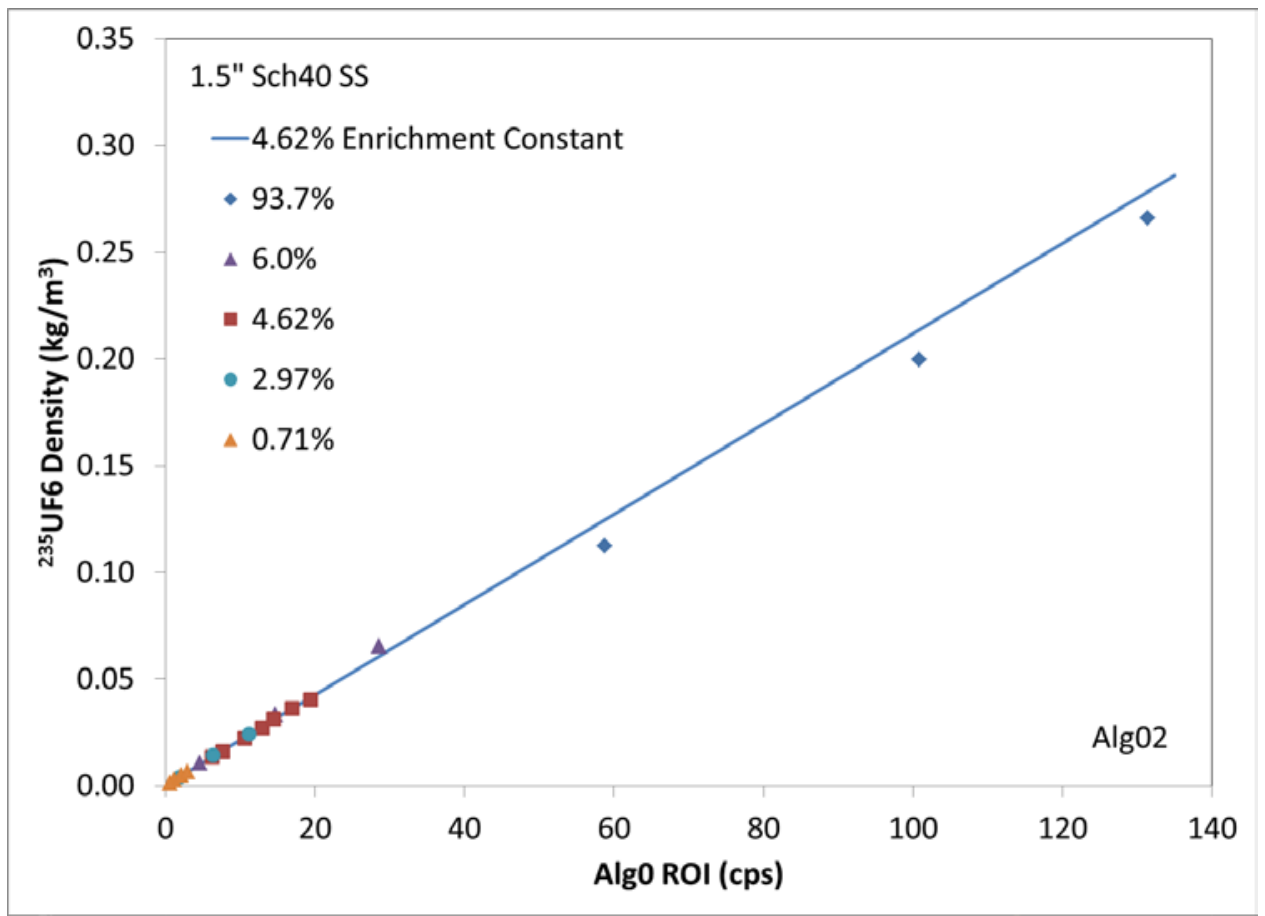

Figure 33. Verification of enrichment constant calculation for Alg02, including the $93.7 \%$ data set 


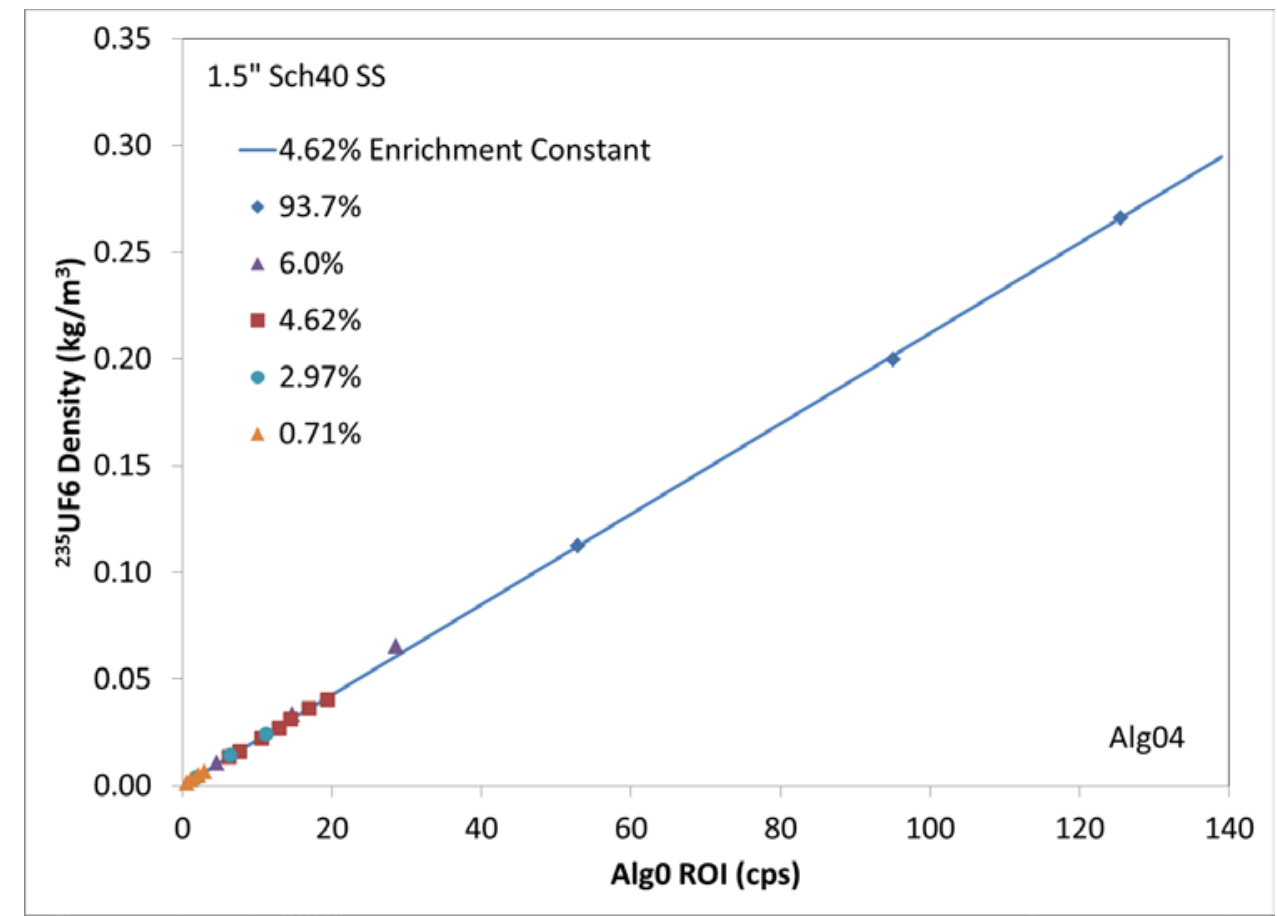

Figure 34. Verification of enrichment constant calculation for Alg04, including the 93.7\% data set

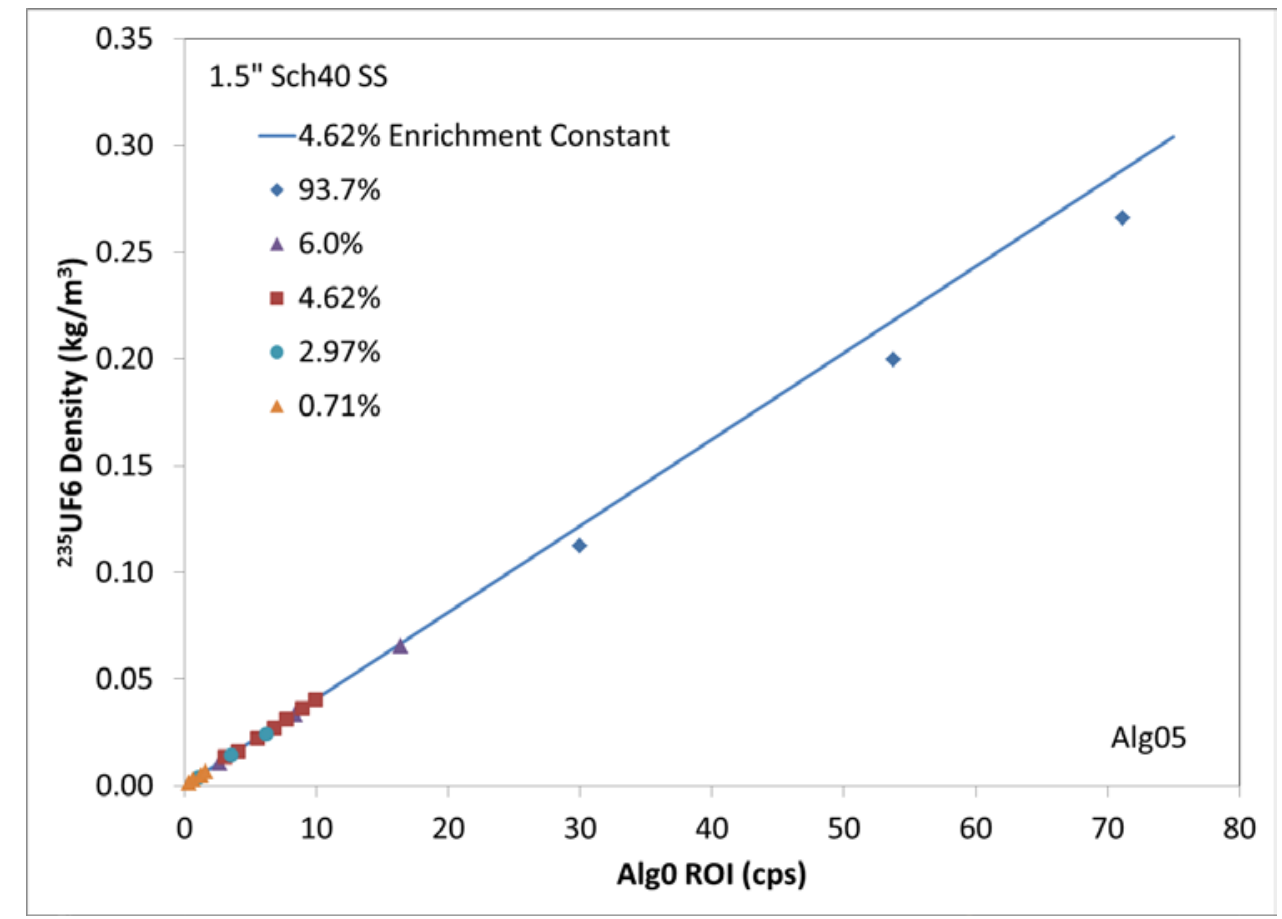

Figure 35. Verification of enrichment constant calculation for Alg05, including the $93.7 \%$ data set 


\subsection{CONCLUSIONS}

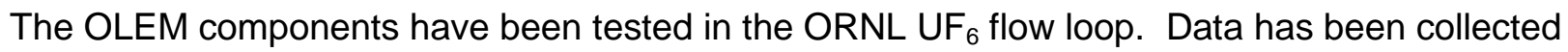
at five different enrichment levels $(0.71 \%, 2.97 \%, 4.62 \%, 6.0 \%$, and $93.7 \%)$ at several pressure conditions. The test data has been collected in the standard OLEM N.4242 file format for each of the conditions with a 10-minute sampling period and then averaged over the span of constant pressures.

Analysis of the collected data has provided enrichment constants that can be used for 1.5" stainless steel schedule 40 measurement sites. The enrichment constant is consistent among all the wide range of enrichment levels and pressures used.

The recommended algorithm settings, including $\mathrm{ROI}$ ranges are provided in Table 3 through Table 8. The best estimate enrichment constants are provided in Table 9. 